\title{
Causal properties of AdS-isometry groups II: BTZ multi-black-holes
}

\author{
Thierry Barbot
}

CNRS, UMR 5669, Ecole Normale Supérieure de Lyon, 46 allée d'Italie, 69364 Lyon, France

Thierry.Barbot@umpa.ens-lyon.fr

\begin{abstract}
This paper is the continuation of [7]. We essentially prove that the family of strongly causal spacetimes defined in [7] associated to generic achronal subsets in $\operatorname{Ein}_{2}$ contains all the examples of BTZ multi-blackholes. It provides new elements for the global description of these multiblack-holes. We also prove that any strongly causal spacetime locally modeled on the anti-de Sitter space admits a well-defined maximal strongly causal conformal boundary locally modeled on $\operatorname{Ein}_{2}$.
\end{abstract}

\section{Introduction}

In [7], we studied certain aspects of causal properties of AdS-spacetimes, i.e., lorentzian manifolds of dimension 3 with negative constant curvature, in other words, locally modeled on the anti-de Sitter space AdS. In particular, we made a detailed analysis on the causality notion in AdS which, to be meaningful, has to be understood as the projection of the causality relation in the universal covering $\widehat{\mathrm{AdS}}$. The Einstein universes $\operatorname{Ein}_{3}$ and $\operatorname{Ein}_{2}$ play

e-print archive: http://lanl.arXiv.org/abs/math.GT/0510065 
an important role in this study, the first one containing a conformal copy of AdS, the second being the conformal boundary of AdS (see $[7, \S 4]$ ).

We also introduced the notion of (generic) closed achronal subset of the conformal boundary $\operatorname{Ein}_{2}($ see $[7, \S 5])$. We associate to it the invisible domains $E(\Lambda) \subset$ AdS and $\Omega(\Lambda) \subset \operatorname{Ein}_{2}$ (Section 8). One of the main results of [7] was the following: assume that $\Lambda$ is non-elementary, and that a torsion-free discrete group of isometries of AdS preserves $\Lambda$. Then, the action of $\Gamma$ on $E(\Lambda)$ is free, properly discontinuous, and the quotient space $M_{\Lambda}(\Gamma)=\Gamma \backslash E(\Lambda)$ is strongly causal (Theorem 10.1). We observed that this construction provides, when $\Lambda$ is a topological circle, the entire family of maximal globally hyperbolic AdS-spacetimes admitting a Cauchy-complete Cauchy surface (Section 11; the same result is obtained in [9] with a different proof). We also discussed in which condition a discrete group of isometries $\Gamma$ is admissible, i.e., preserves a closed, achronal subset as above: essentially, up to a permutation of space and time, admissible groups are precisely the positively proximal groups which are precisely the groups preserving a proper, closed, convex domain of $\mathbb{R} P^{3}$ (see [7, Proposition 10.23]). We also stressed out that there is a natural 1-1 correspondence between admissible groups and pairs of (marked) complete hyperbolic metrics on the same surface (not necessarily closed). Finally, if $\Gamma$ is admissible and non-abelian, there exists a unique minimal, closed, generic achronal $\Gamma$-invariant subset $\Lambda(\Gamma)$ which is contained in every closed achronal $\Gamma$-invariant subset (see [7, Theorem 10.13, Corollary 10.14]). Hence, following the classical terminology used for isometry groups of $\mathbb{H}^{n}$, it is natural to call $\Lambda(\Gamma)$ the limit set of $\Gamma$.

The present paper completes this study in the elementary case, i.e., the case where $\Lambda$ is contained in the past or future of one point in $\operatorname{Ein}_{2}$. In particular, we give the description of invisible domains from elementary, generic, achronal domains (Section 3).

There is another equivalent definition of invisible domains $E(\Lambda(\Gamma))$ when $\Lambda(\Gamma)$ is the limit set of $\Gamma$ (since $\Lambda(\Gamma)$ is minimal, $E(\Lambda(\Gamma)$ ) is in some way maximal among the $\Gamma$-invariant invisible domains). For any element $\gamma$ of $\Gamma$ we define the standard causal domain $C(\gamma)$ as the set of points $x$ in AdS which are not causally related to their image $\gamma x$. The interior of the intersection of all the $C(\gamma)$ is then the standard causal domain of $\Gamma$, denoted by $C(\Gamma)$. Every element $\gamma$ is also the time 1 of a Killing vector field. We then define the absolute causal domain $D(\gamma)$ as the domain of AdS where this Killing vector field has positive norm. The interior of the intersection of all the $D(\gamma)$ is the absolute causal domain $D(\Gamma)$ of $\Gamma$. According to Theorem 8.2 and Corollary 8.4, if $\Gamma$ is a non-cyclic admissible group, then $D(\Gamma)$ and $C(\Gamma)$ both coincide with $E(\Lambda(\Gamma))$. A first version of this theorem, in a very particular case, appears in $[3, \S 7]$. 
But, most of all, we prove that the spacetimes $M_{\Lambda}(\Gamma)$ form a global family of BTZ multi-black-holes, containing and elaborating all the previous examples $[1-5,10,11]$, except the non-static single BTZ black-holes themselves (see Remark 10.3).

In order to develop this assertion, since we do not assume any acquaintance of the reader with this notion, we need some preliminary discussion about black-holes.

\subsection{A quick insight into Schwarzschild and Kerr black-holes}

An honest introduction to the notion of black-hole requires a minimal historical exposition. First of all, a black-hole is a lorentzian manifold $(M, g)$ solution of the Einstein equation: ${ }^{1}$

$$
\operatorname{Ric}_{g}-\frac{R}{2} g=\Lambda \cdot g,
$$

where $\operatorname{Ric}_{g}$ is the Ricci tensor, $R$ is the scalar curvature, i.e., the trace of the Ricci tensor (relatively to the metric $g$ ), and $\Lambda$ is a prescribed real number, the cosmological constant.

It was soon realized by Schwarzschild that this equation with $\Lambda=0$ admits the following 1-parameter family of solutions:

$$
d s^{2}=-\left(1-\frac{2 M}{r}\right) d t^{2}+\frac{d r^{2}}{1-2 M / r}+r^{2} d s_{0}^{2} .
$$

In this expression, $d s_{0}^{2}=d \theta^{2}+\sin ^{2} \theta d \phi^{2}$ is the usual round metric on the sphere $\mathbb{S}^{2}$. The term $r$ is $\sqrt{x^{2}+y^{2}+z^{2}}$, where $(x, y, z, t)$ are coordinates of $\mathbb{R}^{4}$, and $(\theta, \phi)$ are the spherical coordinates of $\left(\frac{x}{r}, \frac{y}{r}, \frac{z}{r}\right) \in \mathbb{S}^{2}$.

Moreover, in some manner, the Schwarzschild metric is the 'unique' solution of the Einstein equation on $\mathbb{R}^{4}$ with spherical symmetry, i.e., invariant by the usual $\mathrm{SO}(3, \mathbb{R}$ )-action (Birkhoff Theorem, see [21] for a more rigorous and detailed exposition). Hence, this metric suits perfectly the role of the model of a non-rotating isolated object (for example, a star) with mass $M$. But these solutions contain a psychological difficulty: the radius of the object could be inferior to $r=2 M$, in which case the singularity $r=2 M$ of the metric cannot be dropped out by considering that it is hidden inside the object, where the general relativity theory does not apply anymore. It has

\footnotetext{
${ }^{1}$ The full Einstein equation contains an additional term on the right side, which is a symmetric trace-free tensor describing the matter and physical forces in the spacetime. The equation stated here is an equation in the void.
} 
been realized that the singularity is not a real one: it appears only in reason of the selected coordinate system. Actually, the Schwarzschild metric on $\mathbb{R}^{4} \backslash\{r=0,2 M\}$ can be isometrically embedded in another lorentzian manifold $M_{\mathrm{KS}}$, diffeomorphic to $\mathbb{R}^{2} \times \mathbb{S}^{2}$, and maximal: $M_{\mathrm{KS}}$ cannot be isometrically embedded in a bigger spacetime. This maximal spacetime is usually described in the following way: equip $\mathbb{R}^{2} \times \mathbb{S}^{2}$ with coordinates $(u, v, \theta, \phi)$ the Kruskal-Szekeres coordinates - where $(\theta, \phi)$ are still the spherical coordinates. Then $M_{\mathrm{KS}}$ is the open domain $U \times \mathbb{R}^{2}$, where $U=\left\{v^{2}-u^{2}<1\right\}$, equipped with the metric

$$
d s_{\mathrm{KS}}^{2}=\left(32 M^{3} / r\right) e^{-r / 2 M}\left(-d v^{2}+d u^{2}\right)+r^{2} d s_{0}^{2} .
$$

The term $r$, defined by $(r / 2 M-1) e^{r / 2 M}=u^{2}-v^{2}$ coincides with the $r$-coordinate on the Schwarzschild domain: hence, the 'singularity' $r=2 M$ vanishes; it corresponds to the locus $\left\{u^{2}=v^{2}\right\}$.

The domain $O=\left\{0<u^{2}-v^{2}<1\right\}$ is the outer domain: it is thought as the region where the typical (prudent) observer takes place. There are two other regions: $B^{ \pm}=\left\{u^{2}-v^{2}<0, \pm v>0\right\}$. Moreover, $M_{\mathrm{KS}}$ is time-oriented so that $v$ increases with time.

$B^{+}$enjoys the following remarkable property: there is no future-oriented causal curve starting from a point in $B^{+}$and reaching a point in $O$. Similarly, a past-oriented causal curve cannot escape from $B^{-}$. In a more "physical" language, photons cannot escape from $B^{+}: B^{+}$is invisible from $O$. In other words, $B^{+}$is a black-hole. The no-return frontier $\{v=|u|\}$ is the (event) horizon.

The family of Schwarzschild metrics is actually included in a more general family of solutions, the Kerr metrics:

$$
\begin{aligned}
& -\left(1-\frac{2 M r}{\rho^{2}}\right) d t^{2}+\frac{\rho^{2}}{\Delta} d r^{2}-\sin ^{2}(\theta) \frac{4 M r a}{\rho^{2}} d \phi d t+\rho^{2} d \theta^{2} \\
& +\left(r^{2}+a^{2}+\sin ^{2}(\theta) \frac{2 M r a}{\rho^{2}}\right) \sin ^{2}(\theta) d \phi^{2}
\end{aligned}
$$

where $\Delta=r^{2}-2 M r+a^{2}, \mathrm{t} \rho^{2}=r^{2}+a^{2} \cos ^{2}(\theta), M$ is positive (the "mass"), and $a$ is a real number (the "angular momentum per mass unit").

A phenomenon similar to the embedding of Schwarzschild spacetime in Kruskal-Szekeres coordinates still applies: every Kerr-spacetime can be embedded in a natural way into a maximal spacetime $M_{\mathrm{Kerr}}^{\max }$ where the singularities appearing in the Kerr-coordinates vanish, and where some domains 
deserve the appellation "black-hole". But the description of this completion is much more involved: it is the entire matter of the excellent book [22], which is also one of the best references for a rigorous description of Schwarzschild black-holes.

\subsection{Towards a general definition of black-holes}

The basic facts on Schwarzschild spacetimes presented above are sufficient to provide a quite satisfactory illustration of essential notions that should appear in any mathematical definition of black-holes. Let us be more precise and express some problems arising when trying to elaborate such a general theory, i.e., to specify what are the spacetimes deserving the appellation of spacetime with black-hole.

(1) Where are the typical observers? The description of the Schwarzschild black-hole makes clear that this notion is relative to the region $O$ where the observers are assumed to stay. A black-hole is simply a connected component of the region invisible from $O$. How to define the region $O$ in $M$ without specifying the black-hole itself?

(2) Is some part of $M$ missing? The notion of black-hole is a global property, depending on the entire $M$. For example, if we delete from the Minkowski space some regions, we can easily produce in an artificial way regions invisible from the observers (assuming solved the first question above), which does not correspond to a physically relevant example of black-hole. Hence, we have to define a notion of "full" spacetime ensuring that some part of the invisible domain is not simply due to the absence of some relevant region.

Remark 1.1. To these two basic problems, another important requirement, traditionally appearing in the physical literature, should be added: the Cosmic Censorship. This condition admits many different formulations. It is most of the time expressed in the form "singularities of the spacetime must be hidden to the observers by the horizons". There is a geometrical way to translate this notion, interpreting singularities as final points of non-complete causal geodesics or as causal singularities: we could define spacetimes satisfying the Cosmic Censorship if causal closed curves, if any, all belong to a black hole, and if any non-complete causal future-oriented ray in $M$, with starting point visible from one observer, must enter a black-hole.

There is a particular case of the Cosmic Censorship conjecture often stated as follows: "generically" (to be defined!), maximal globally hyperbolic spacetimes are maximal, i.e., cannot be embedded in a bigger spacetime, even not globally hyperbolic. But the spacetimes containing a black-hole we will 
consider here are not globally hyperbolic, hence this expression of Cosmic Censorship is not relevant for them.

Some answer to the first question above seems to be widely accepted: observers lie near the conformal boundary. Then, we can define the blackholes as the connected components of the invisible domain, i.e., the interior of the region of $M$ containing the points $x$ such that no causal future-oriented curve starting from $x$ tends to a point in the conformal boundary of $M$. Of course, this solution arises immediately another question: what is the conformal boundary? This new question is not answered in full generality for a nice recent survey on related questions see [15]. We can summarize many works by observing that this point is most of the time solved simply by prescribing from the beginning what is the conformal boundary, without making sure that the proposed boundary is maximal in any meaning. ${ }^{2}$ However, in our special AdS context, we have a completely satisfactory and easy definition of conformal boundary of strongly causal AdS spacetimes (see next Section).

Concerning the second question, encouraged by the examples of KruskalSzekeres and Kerr spacetimes, one could hope that a good answer is simply to require $M$ to be maximal in the sense that it cannot be isometrically embedded in a bigger spacetime satisfying the Einstein equation. Unfortunately, this attempt fails for BTZ black-holes: they are not maximal in this meaning. Furthermore, such a definition of full would lead to some incoherence in the maximal Kerr spacetime. The restriction to causal spacetimes, i.e., to require that $M$ is a causal spacetime and that it cannot be embedded in a bigger causal spacetime does not solve the problem: once more, it does not apply to BTZ black-holes (see Remark 10.7).

Our deceiving conclusion is that we still do not know how to express in a satisfying way what is a good notion of full spacetime, even in the AdS-background. As a positive element of answer, we stress out that all the examples reproduced later enjoy the following properties of 'maximal' nature: every connected component of their conformal boundary is maximal globally hyperbolic, and every black-hole, i.e., every connected component of the invisible domain (but this domain will always be connected) is maximal globally hyperbolic too (see Remark 10.9). Observe that these requirements (global hyperbolicity of black-holes and observer-spacetime) reflect in some way the Cosmic Censorship principle for globally hyperbolic spacetimes (Remark 1.1).

\footnotetext{
${ }^{2}$ Anyway, for the Kruskal-Szekeres spacetimes $M_{\mathrm{KS}}$ and maximal Kerr-spacetimes $M_{\mathrm{Kerr}}^{\max }$, which are all asymptotically flat, the conformal boundary is well defined in a fully satisfying way.
} 


\subsection{BTZ black-holes}

First of all, BTZ-multi-black-holes have dimension $2+1$, i.e., are 3 -dimensional. In this low dimension the Einstein equation is remarkably simplified: the solutions have all constant sectional curvature with the same sign that the cosmological constant $\Lambda$ has. We only consider the case $\Lambda<0$. Hence, up to rescaling, the spacetimes satisfying the Einstein equation are precisely the spacetimes locally modeled on AdS.

This special feature allows us to propose a correct answer to the first question in the preceding paragraph, in the $2+1$-dimensional case, i.e., with AdS-background, due to the natural conformal completion of AdS by $\operatorname{Ein}_{2}$. More precisely, the spacetimes $M$ we will consider are locally modeled in AdS - in short, they are AdS-spacetimes. Hence, their universal covering admits a development $\mathcal{D}: \widetilde{M} \rightarrow$ AdS. Define then the lifted conformal boundary $\Omega$ as the interior of the set of final extremities in $\operatorname{Ein}_{2}=\partial \mathrm{AdS}$ of future-oriented causal curves with relative interior contained in $\mathcal{D}(\widetilde{M})$. We could try to define the conformal boundary of $M$ as the quotient of $\Omega$ by the holonomy group $\rho(\Gamma)$. Unfortunately, this definition admits an uncomfortable drawback: the development $\mathcal{D}$ could be non-injective, which requires a modification of the definition above. Moreover, we have no guarantee a priori that the action of $\rho(\Gamma)$ on $\Omega$ is free and proper.

In Section 9, we show how to solve these difficulties in the context of strongly causal spacetimes: every strongly causal AdS-spacetime admits a natural strongly causal conformal boundary (Theorem 9.5).

For that reason (besides the physical coherence of such an assumption) we restrict from now to strongly causal spacetimes. Now we can state our peculiar definition:

Definition 1.2. An AdS-spacetime with black hole is a strongly causal AdS spacetime $M$ such that:

- $M$ admits a non-empty strongly causal conformal boundary $O$,

- the past of $O$, i.e., the region of $M$ made of initial points of futureoriented causal curves ending in $O$, is not the entire $M$.

Every connected component of the interior of the complement in $M$ of the past of $O$ is a black-hole.

Let us now collect the examples. While considering discrete groups of isometries of AdS, we have constructed many examples of spacetimes locally 
modeled on AdS: the quotients $M(\Gamma)=\Gamma \backslash E(\Gamma)$, where $\Gamma$ is a non-abelian torsion-free discrete admissible subgroup of Isom(AdS). Every $M(\Gamma)$ contains a black-hole, except if it is globally hyperbolic, i.e., except if $\Lambda$ is a topological circle.

This construction applies in more cases, even if $\Gamma$ is abelian, but the elementary achronal subset $\Lambda$ must be selected: it must contain at least two points, and $\Gamma$ must be a cyclic subgroup, generated by an isometry $\gamma_{0}$. More precisely:

- The conical case: This is the case where $\Lambda$ is the union of two lightlike geodesic segments $[y, x],[z, x]$, each of them not reduced to single point and with a common extremity $x$, which is their common past. Then $\gamma_{0}$ must be a hyperbolic-hyperbolic element (see Definition 4.2), and $y, z$ must be attractive fixed points of $\gamma_{0}$.

- The splitting case: This is the case where $\Lambda$ is a pair of non-causally related points in $\operatorname{Ein}_{2}$. As in the previous case, these points must be the attractive and repulsive fixed points of the hyperbolic-hyperbolic element $\gamma_{0}$.

- The extreme case: This last case is the case where $\Lambda$ is a light-like segment, not reduced to a single point. Then, $\gamma_{0}$ must be a parabolichyperbolic element (see Definition 4.2) fixing the two extremities of $\Lambda$.

In all these cases, the quotient $M_{\Lambda}(\Gamma)=\Gamma \backslash E(\Lambda)$ is still a spacetime with conformal boundary, and with non-empty invisible set: a black-hole.

Since it requires a basic knowledge of AdS geometry, the description of these spacetimes is postponed to Section 10. We just mention in this introduction that they essentially ${ }^{3}$ include all the previously examples named BTZ black-holes $([1-5,10,11])$, but also contains new examples: mainly, the case where $\Gamma$ is not finitely generated, and also the conical case, which is not considered as a spacetime with a black-hole in these references, probably because it is obviously non-maximal since, in this case, $M_{\Lambda}(\Gamma)$ embeds isometrically into $M_{y z}(\Gamma)$.

\subsection{Notation}

This paper should be read jointly with [7], in which all basic notions and notation are introduced. Let us recall some of them, for the reader's convenience:

\footnotetext{
${ }^{3}$ Except the particular case of non-static single BTZ black-holes; see Remark 10.3.
} 
- AdS is the anti-de Sitter space, i.e., the locus $\{Q=-1\}$ in $E \approx \mathbb{R}^{2,2}$ equipped with a quadratic form $Q$ of signature $(2,2)$. It is isometric to the Lie group $\mathrm{SL}(2, \mathbb{R})$ endowed with the lorentzian metric defined by the Killing form. Its universal covering is denoted by $\widetilde{\mathrm{AdS}}$ and its projection into the sphere $S(E)$ of rays in $E$, the Klein model, is denoted by $\mathbb{A D S}$. The projective Klein model $\overline{\mathbb{A D S}}$ is the projection in the projective space $P(E)$. It is naturally identified with the Lie group $G=\operatorname{PSL}(2, \mathbb{R})$. All these lorentzian manifolds are oriented and chronologically oriented.

- A spacetime is a lorentzian manifold which is oriented and chronologically oriented. An AdS-spacetime is a spacetime locally modeled on the anti-de Sitter space AdS.

- Affine domains in AdS, $\widetilde{\mathrm{AdS}}, \mathbb{A D S}$ are lifts of affine domains in $\overline{\mathbb{A D S}}$, i.e., intersection in $P(E)$ between $\overline{\mathbb{A D S}}$ and affine domains $U$ of $P(E)$ such that the intersection between the projective plane $\partial U$ and $\overline{\mathbb{A D S}}$ is a space-like surface; it is then an isometric copy of the hyperbolic plane $\mathbb{H}^{2}$.

- The isometry group of $\overline{\mathrm{ADS}}$ is $G \times G$. The isometry group of $\widetilde{\mathrm{AdS}}$ is isomorphic to $\widetilde{G} \times \widetilde{G}$ quotiented by the cyclic diagonal group generated by $(\delta, \delta)$, where $\delta$ is a generator of the center of $\widetilde{G}$, the universal covering of $G$.

- There is a conformal embedding of AdS into the Einstein universe $\operatorname{Ein}_{3}$, which is $\mathbb{S}^{2} \times \mathbb{S}^{1}$ equipped with (the conformal class of) the lorentzian metric $d s^{2}-d t^{2}$, where $d s^{2}$ is the round metric on the 2-sphere and $d t^{2}$ the usual metric on $\mathbb{S}^{1} \approx \mathbb{R} / 2 \pi \mathbb{Z}$. The universal covering of $\operatorname{Ein}_{3}$ is denoted by $\widehat{\operatorname{Ein}}_{3}$. There is a conformal embedding of $\widehat{\operatorname{AdS}}$ into $\widehat{\operatorname{Ein}}_{3}$. The boundary of the image of this embedding is the conformal boundary $\partial \widehat{\mathrm{AdS}} \approx \widehat{\operatorname{Ein}}_{2}$. The projection $\overline{\operatorname{Ein}}_{2}$ of $\operatorname{Ein}_{2}$ in $P(E)$ is the conformal boundary of $\overline{\mathrm{ADS}}$.

- $\overline{\operatorname{Ein}}_{2}$ is bifoliated by two transverse foliations $\overline{\mathcal{G}}_{\mathrm{L}}, \overline{\mathcal{G}}_{\mathrm{R}}$. Every leaf of $\overline{\mathcal{G}}_{\mathrm{L}}$ (the left foliation) or of $\overline{\mathcal{G}}_{\mathrm{R}}$ (the right foliation) is a light-like geodesic, canonically isomorphic to the real projective line $\mathbb{R} P^{1}$. Every leaf of $\overline{\mathcal{G}}_{\mathrm{L}}$ intersects every leaf of $\overline{\mathcal{G}}_{\mathrm{R}}$ at one and only one point. Finally, the leaf space of the left (respectively right) foliation is naturally isomorphic to the real projective line. It is denoted by $\mathbb{R} P_{\mathrm{L}}^{1}$ (respectively $\mathbb{R} P_{\mathrm{R}}^{1}$ ).

- For all causality notions, we refer to [8]. In [7] we also present the causality notions pertinent for our purpose. An achronal subset of $\widehat{\operatorname{Ein}}_{2}$ is a subset $\widetilde{\Lambda}$ such that for any pair $(x, y)$ of distinct elements of $\widetilde{\Lambda}, x$ and $y$ are not causally related in $\widehat{\operatorname{Ein}}_{2}$. An achronal subset $\widetilde{\Lambda}$ is pure light-like if it contains two opposite elements $x, \delta(x)$; if not, it is generic. A generic achronal subset is elementary if it is contained in the union $l \cup l^{\prime}$, where $l, l^{\prime}$ are light-like geodesic segments in $\widehat{\operatorname{Ein}}_{2}$ 
(see $[7, \S 8.7])$. All these notions project on similar notions in $\operatorname{Ein}_{2}$. See $[7, \S 5]$, for more details.

- The invisible domain of a generic, closed, achronal subset $\widetilde{\Lambda}$ in $\widehat{\operatorname{Ein}}_{2}$ is the set of points in $\widehat{\operatorname{Ein}}_{2}$ which are not causally related to any element of $\widetilde{\Lambda}$. It is denoted by $\Omega(\widetilde{\Lambda})$. It is the region between the graphs $\widetilde{\Lambda}^{+}$ and $\widetilde{\Lambda}^{-}$of two 1-Lipschitz functions from $\mathbb{S}^{1}$ into $\mathbb{R}$ (see $[7, \S 8.6]$ ). The invisible domain $E(\widetilde{\Lambda})$ in $\widetilde{\mathrm{AdS}}$ is the set of points in $\widetilde{\mathrm{AdS}} \subset \widehat{\operatorname{Ein}}_{3}$ which are not causally related to any element of $\widetilde{\Lambda}$ in $\widehat{\operatorname{Ein}}_{3}$.

\section{Strongly causal spacetimes}

A lorentzian manifold $M$ is strongly causal if for every point $x$ in $M$ every neighborhood of $x$ contains an open neighborhood $U$ which is causally convex, i.e., such that any causal curve in $M$ joining two points in $U$ is actually contained in $U$ (see $[7, \S 2.3]$ ). An isometric action of a group $\Gamma$ on a strongly causal lorentzian manifold $M$ is strongly causal if any point $x$ of $M$ admits arbitrarily small neighborhoods $U$ such that for every non-trivial element $\gamma$ of $\Gamma$ no point of $U$ is causally related to a point of $\gamma U$. Clearly, if $\Gamma$ is a group of isometries of a strongly causal lorentzian manifold $M$ acting freely and properly discontinuously, the quotient manifold $\Gamma \backslash M$ is strongly causal if and only if the action of $\Gamma$ is strongly causal.

In this paper we need to prove that certain isometric actions are strongly causal. In this section we provide two lemmas useful for this purpose.

In the first lemma we consider a strongly causal lorentzian manifold $M$ admitting a Killing vector field $X$. We assume that $X$ is everywhere spacelike. Let $\phi^{t}$ be the flow generated by $X$ and assume that the $\mathbb{R}$-action defined by $\phi^{t}$ is free and properly discontinuous. Let $Q^{\phi}$ be the orbit space of $\phi^{t}$ and $\pi: M \rightarrow Q^{\phi}$ the projection map. It is easy to show that $\pi$ is a locally trivial fibration with fibers homeomorphic to $\mathbb{R}$. For every tangent vector $v$ of $Q^{\phi}$ at a point $y$ let $x$ be any element of $\pi^{-1}(y)$ and let $w$ be the unique tangent vector to $M$ at $x$ orthogonal to $X(x)$ and such that $d \pi_{x}(w)=v$. Since $\phi^{t}$ is isometric the norm of $w$ does not depend on the choice of $x$ : we define the norm $|v|$ as this norm $|w|$. This procedure defines a lorentzian metric on $Q^{\phi}$.

Lemma 2.1. If the lorentzian manifold $Q^{\phi}$ is strongly causal then the action of $\phi^{1}$ on $M$ is strongly causal.

Proof. Let $x$ be any element of $M$ and $U$ any neighborhood of $x$. Let $y=$ $\pi(x)$. Since $Q^{\phi}$ is assumed strongly causal, $U^{\prime}=\pi(U)$ contains a causally 
convex open neighborhood $V^{\prime}$ of $y$. Define $V=\pi^{-1}\left(V^{\prime}\right)$ : this open subset is diffeomorphic to $V^{\prime} \times \mathbb{R}$, such that the restriction of $\phi^{t}$ to $V$ corresponds to the translation on the $\mathbb{R}$-factor. We also take the convention that the $\mathbb{R}$ coordinate of $x$ is zero: $x \approx(y, 0)$. Shrinking $V^{\prime}$ if necessary, we can assume that the space-like norm of $X$ on $V$ is uniformly bounded by some constant $C$ and, moreover, that any causal curve in $V^{\prime}$ has lorentzian length less than $1 / 2 C$. It then follows that the $\mathbb{R}$-coordinate of any causal curve in $V$ cannot vary more than $1 / 2$. Let $W$ be a causally convex open neighborhood of $x$ contained in $\left.V^{\prime} \times\right]-1 / 4,+1 / 4[\subset V$. We claim that for every non-zero integer $n$ non-point of $\phi^{n} W$ is causally related to a point of $W$. Indeed, assume by contradiction that there is a causal curve $c: I \rightarrow M$ joining an element $(z, t)$ to an element $\left(z^{\prime}, s+n\right)$ with $z, z^{\prime}$ in $V^{\prime}$ and $s, t$ in $]-1 / 4,+1 / 4[$. The projection $\pi \circ c$ is a causal curve in $Q$ joining $\pi(z), \pi\left(z^{\prime}\right)$. Since $V^{\prime}$ is causally convex $\pi \circ c$ is contained in $V^{\prime}$, i.e., $c$ is contained in $V$. Hence the $\mathbb{R}$-coordinate cannot vary along $c$ more than $1 / 2$. We obtain a contradiction since $s+n-t$ has absolute value bigger than $1 / 2$.

Remark 2.2. Lemma 2.1 remains true when the action of $\phi^{t}$ is not free but periodic. In this case the group generated by $\phi^{1}$ is finite. Details are left to the reader.

We will apply Lemma 2.1 in the case where $M$ has dimension 3 and is simply connected. In this situation the map $\pi$ is a fibration, and the homotopy sequence of this fibration, implies that $Q^{\phi}$ is a 2-dimensional manifold which is simply connected. Hence a very nice complement is

Lemma 2.3. Any simply connected 2-dimensional lorentzian manifold is strongly causal.

Proof. Any simply connected surface is diffeomorphic to the sphere or the plane $\mathbb{R}^{2}$. Since the sphere does not admit any lorentzian metric we just have to consider the case of the plane $\mathbb{R}^{2}$. Any reader acquainted with dynamical systems will recognize that the lemma follows quite easily from the Poincaré-Bendixon Theorem applied to the light-like foliations: every leaf of one of these foliations is a closed embedding of the real line in $\mathbb{R}^{2}$ and for any point $x$ in $\mathbb{R}^{2}$ the future (respectively the past) of $x$ is the domain bounded by $r_{1} \cup r_{2}$, $\mathrm{m}$ where $r_{1}, r_{2}$ are the future-oriented (respectively past oriented) light-like geodesic rays starting from $x$. Details are left to the readers. For readers who are still not convinced, we refer to $[8$, Theorem 3.43] where a slightly better statement is proved: lorentzian metrics on the 2-plane are stably causal, which is stronger than being strongly causal. 


\section{Description of the elementary generic achronal subsets of $\widehat{\operatorname{Ein}}_{2}$}

In this section, we complete the descriptions of invisible domains $E(\widetilde{\Lambda})$ and $\Omega(\widetilde{\Lambda})$ by considering the elementary cases. For the reader's convenience, we start with the splitting case, which is already described in $[7, \S 8.8]$.

\subsection{The splitting case}

It is the case $\widetilde{\Lambda}=\{x, y\}$, where $x, y$ are two non-causally related points in $\widehat{\operatorname{Ein}}_{2}$. Then $\{x, y\}$ is a gap pair, and there are two associated ordered gap pairs that we denote respectively by $(x, y)$ and $(y, x)$ (see [7, Definition 8.24]). $\widetilde{\Lambda}^{+}$is the union $\mathcal{T}_{x y}^{+} \cup \mathcal{T}_{y x}^{+}$of two future-oriented light-like segments with extremities $x, y$ that we call upper tents. Such an upper tent is the union of two light-like segments, one starting from $x$, the other from $y$, and stopping at their first intersection point, that we call the upper corner.

Similarly, $\widetilde{\Lambda}^{-}$is the union $\mathcal{T}_{x y}^{-} \cup \mathcal{T}_{y x}^{-}$of two lower tents admitting a similar description, but where the light-like segments starting from $x, y$ are now past-oriented (see figures 1 and 2), and sharing a common extremity, the lower corner.

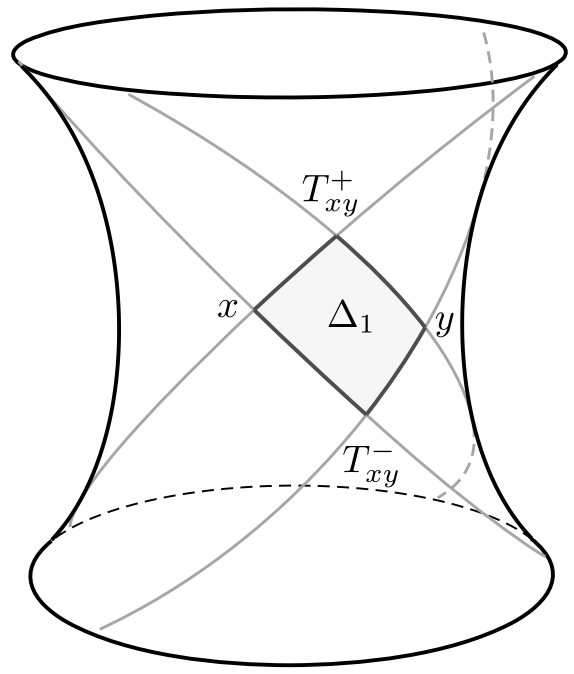

Figure 1: Upper and lower tents. 


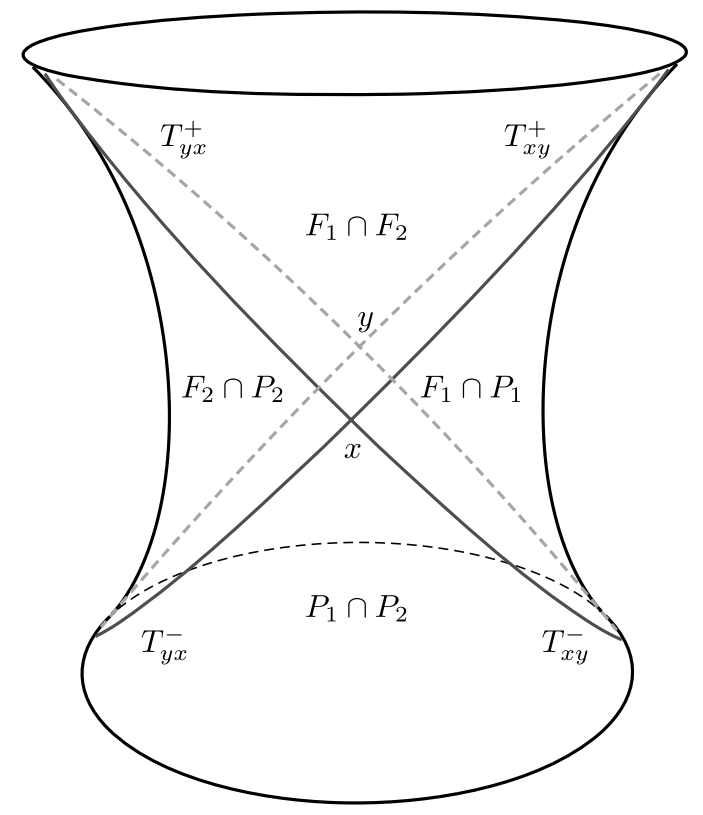

Figure 2: The splitting case. The domain $E(\Lambda)$ is between the hyperplanes $x^{\perp}$ and $y^{\perp}$. These hyperplanes, tangent to the hyperboloid, are not drawn, except their intersections with the hyperboloid, which are the upper and lower tents $\mathcal{T}_{x y}^{ \pm}, \mathcal{T}_{y x}^{ \pm}$.

The invisible domain $\Omega(\widetilde{\Lambda})$ from $\widetilde{\Lambda}$ in $\widehat{\operatorname{Ein}}_{2}$ is the union of two diamondshaped regions $\widetilde{\Delta}_{1}, \widetilde{\Delta}_{2}$. The boundary of $\widetilde{\Delta}_{1}$ is the union $\mathcal{T}_{x y}^{+} \cup \mathcal{T}_{x y}^{-}$, and the boundary of $\widetilde{\Delta}_{2}$ is $\mathcal{T}_{y x}^{+} \cup \mathcal{T}_{y x}^{-}$. We project the entire picture in some affine region $V \approx \mathbb{R}^{3}$ of $S(E)$ such that:

- $V \cap \mathbb{A D S}$ is the interior of the hyperboloid: $\left\{x^{2}+y^{2}<1+z^{2}\right\}$,

$-\Lambda=\{(1,0,0),(-1,0,0)\}$.

Then, $E(\Lambda)$ is the region $\{-1<x<1\} \cap \mathbb{A D S}$. One of the diamond-shaped regions $\widetilde{\Delta}_{i}$ projects to $\Delta_{1}=\left\{-1<x<1, y>0, x^{2}+y^{2}=1+z^{2}\right\}$, the other projects to $\Delta_{2}=\left\{-1<x<1, y<0, x^{2}+y^{2}=1+z^{2}\right\}$. The past of $\Delta_{1}$ in $E(\Lambda)$ is $P_{1}=\{(x, y, z) \in E(\Lambda) / z<y\}$, and the future of $\Delta_{1}$ in $E(\Lambda)$ is $F_{1}=\{(x, y, z) \in E(\Lambda) / z>-y\}$. We have of course a similar description for the future $F_{2}$ and the past $P_{2}$ of $\Delta_{2}$ in $E(\Lambda)$. Observe:

- The intersections $F_{1} \cap F_{2}$ and $P_{1} \cap P_{2}$ are disjoint. They are tetrahedra in $S(E): F_{1} \cap F_{2}$ is the interior of the convex hull of $\Lambda^{+}$, and $P_{1} \cap P_{2}$ is the interior of the convex hull of $\Lambda^{-}$. 
- The intersection $F_{1} \cap P_{1}$ (respectively $F_{2} \cap P_{2}$ ) is the intersection between $\mathbb{A D S}$ and the interior of a tetrahedron in $S(E)$ : the convex hull of $\Delta_{1}$ (respectively $\Delta_{2}$ ).

Definition 3.1. $E^{+}(\Lambda)=F_{1} \cap F_{2}$ is the future globally hyperbolic convex core; $E^{-}(\Lambda)=P_{1} \cap P_{2}$ is the past globally convex core.

This terminology is justified by the following (easy) fact: $F_{1} \cap F_{2}$ (respectively $P_{1} \cap P_{2}$ ) is the invisible domain $E\left(\Lambda^{+}\right)$(respectively $E\left(\Lambda^{-}\right)$). Hence, they are indeed globally hyperbolic.

The intersection between the closure of $E(\Lambda)$ in $S(E)$ and the boundary $\mathcal{Q}$ of $\mathbb{A D S}$ is the union of the closures of the diamond-shaped regions. Hence, $\Delta_{1,2}$ can be thought of as the conformal boundaries at infinity of $E(\Lambda)$. Starting from any point in $E(\Lambda)$ to $\Delta_{i}$ we have to enter in $F_{i} \cap P_{i}$, hence we can adopt the following definition:

Definition 3.2. $F_{1} \cap P_{1}$ is an end of $E(\Lambda)$.

Finally

Definition 3.3. The future horizon is the past boundary of $F_{1} \cap F_{2}$; the past horizon is the future boundary of $P_{1} \cap P_{2}$.

Proposition 3.4. $E(\Lambda)$ is the disjoint union of the future and past globally hyperbolic cores $E^{ \pm}(\Lambda)$, of the two ends, and of the past and future horizons.

Remark 3.5. In the conventions of $[4,5,11]$, the globally hyperbolic convex cores $F_{1} \cap F_{2}$ and $P_{1} \cap P_{2}$ are regions of type $I I$, also called intermediate regions. The ends $F_{1} \cap P_{1}$ and $F_{2} \cap P_{2}$ are outer regions, or regions of type $I$.

\section{$3.2 \quad$ The extreme case}

The extreme case is harder to picture out since $\Omega(\widetilde{\Lambda})$ and $E(\widetilde{\Lambda})$ are not contained in an affine domain (see figure 3). Assume that $y$ is in the future of $x$. Observe that $\widetilde{\Lambda}^{ \pm}$are then pure light-like. Hence, $E\left(\widetilde{\Lambda}^{ \pm}\right)$are empty. The region $\Omega(x, y)$ is a "diamond" in $\operatorname{Ein}_{2}$ (we call it an extreme diamond) admitting as boundary four light-like segments: the segments $[y, \delta(x)],[x, \delta(x)]$, $\left[\delta^{-1}(y), x\right]$, and $\left[y, \delta^{-1}(y)\right]$.

A careful analysis shows that $E(x, y)$ is precisely the intersection between the past and the future of $\Omega(x, y)$.

Keeping $x$ fixed, and considering a sequence $y_{n}$ converging to $y$, with $y_{n}$ non-causally related to $x$, one of the diamond-shaped regions $\Delta_{i}^{n}$ of the 


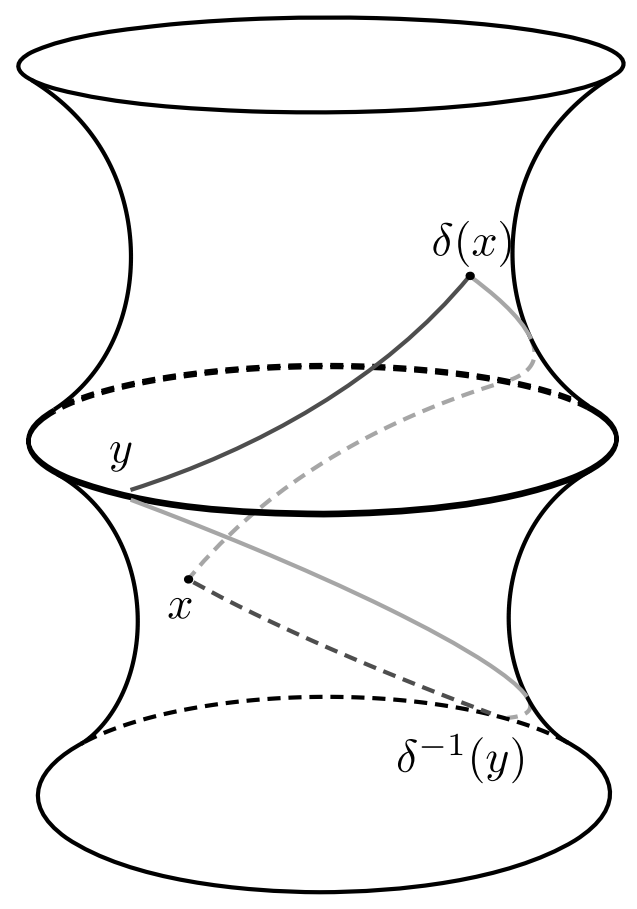

Figure 3: The extreme case. The point $\delta(x)$ is in the front of the upper hyperboloid. The point $x$ is in the rear of the lower hyperboloid. The domain $E(\Lambda)$ is the domain between the hyperplanes $\delta(x)^{\perp}$ and $\delta^{-1}(y)^{\perp}$.

associated $\Omega\left(x, y_{n}\right)$ - let us say, $\Delta_{2}^{n}$ - vanishes. The other converges to the entire region $\Omega(x, y)$. The various parts of the domains $E\left(x, y_{n}\right)$, namely the globally hyperbolic convex cores $E^{ \pm}\left(x, y_{n}\right)$ and the ends, vanish, except one end, which comes closer and closer to the entire $E(x, y)$.

\subsection{The conical case}

In the conical case, $\Lambda$ is an upper or lower tent. By symmetry, we can consider only the upper case: $\Lambda=\mathcal{T}_{x y}^{+}=[x, z] \cup[z, y]$. Then, $\Lambda^{+}=\Lambda \cup \mathcal{T}_{y x}^{+}$, and $\Lambda^{-}$is the pure light-like subset $\Lambda \cup \mathcal{T}_{y x}^{-}$. In the notation of Section 3.1, $\Lambda^{+}$is the future boundary of the diamond $\Delta_{1}$, and $\Omega(\Lambda)$ is the diamond $\Delta_{2}$. Then, in some affine domain, $E(\Lambda)=\{(x, y, z) /-1<x<1, z>y\}$. It can be described as the future in $V$ of $\Delta_{2}$. It is also the intersection between the past of $x$, the past of $y$, and the complement of the past of $z$. Finally, $E(\Lambda)$ is the union of $F_{1} \cap F_{2}, F_{2} \cap P_{2}$, and the component of the past horizon of $E(x, y)$ separating these two regions (see figure 2). 


\section{Causal domains of isometries of AdS}

\subsection{The isometry group}

We recall some facts established in $[7, \S 9]$, concerning isometries preserving generic achronal subsets of $\operatorname{Ein}_{2}$. We use the identification $\overline{\mathbb{A D S}} \approx G=$ $\operatorname{PSL}(2, \mathbb{R})$ (cf. Notation section). Then $\widetilde{\mathrm{AdS}}$ can be identified with the universal covering $\widetilde{G}=\widetilde{\mathrm{SL}}(2, \mathbb{R})$. Denote by $\bar{p}: \widetilde{G} \rightarrow G$ the covering map, and by $Z$ the kernel of $\bar{p}$ : $Z$ is cyclic, it is the center of $\widetilde{G}$. Let $\delta$ be a generator of $Z$ : we select it in the future of the neutral element id.

$\widetilde{G} \times \widetilde{G}$ acts by left and right translations on $\widetilde{G}$. This action is not faithful: the elements acting trivially are precisely the elements in $\mathcal{Z}$, the image of $Z$ by the diagonal embedding. The isometry group $\widehat{\mathrm{SO}}_{0}(2,2)$ is then identified with $(\widetilde{G} \times \widetilde{G}) / \mathcal{Z}$.

Let $\mathcal{G}$ be the Lie algebra $\operatorname{sl}(2, \mathbb{R})$ of $G$ : the Lie algebra of $(\widetilde{G} \times \widetilde{G})_{/ \mathcal{Z}}$ is $\mathcal{G} \times \mathcal{G}$. We assume that the reader is familiar with the notion of elliptic, parabolic, hyperbolic elements of $\operatorname{PSL}(2, \mathbb{R})$. Observe that hyperbolic (respectively parabolic) elements of $\operatorname{PSL}(2, \mathbb{R})$ are the exponentials $\exp (A)$ of hyperbolic (respectively parabolic, elliptic) elements of $\mathcal{G}=\operatorname{sl}(2, \mathbb{R})$, i.e., such that $\operatorname{det}(A)<0$ (respectively $\operatorname{det}(A)=0, \operatorname{det}(A)>0)$.

Definition 4.1. An element of $\widetilde{G}$ is hyperbolic (respectively parabolic, elliptic) if it is the exponential of a hyperbolic (respectively parabolic, elliptic) element of $\mathcal{G}$.

Definition 4.2. An element $\gamma=\left(\gamma_{\mathrm{L}}, \gamma_{\mathrm{R}}\right)$ of $\widetilde{G} \times \widetilde{G}$ is synchronized if, up to a permutation of left and right components, it has one of the following forms:

- Hyperbolic translation: $\gamma_{\mathrm{L}}$ is trivial and $\gamma_{\mathrm{R}}$ is hyperbolic;

- Parabolic translation: $\gamma_{\mathrm{L}}$ is trivial and $\gamma_{\mathrm{R}}$ is parabolic;

- Hyperbolic-hyperbolic: $\gamma_{\mathrm{L}}$ and $\gamma_{\mathrm{R}}$ are both non-trivial and hyperbolic;

- Parabolic-hyperbolic: $\gamma_{\mathrm{L}}$ is parabolic and $\gamma_{\mathrm{R}}$ is hyperbolic;

- Parabolic-parabolic: $\gamma_{\mathrm{L}}$ and $\gamma_{\mathrm{R}}$ are both non-trivial and parabolic;

- Elliptic: $\gamma_{\mathrm{L}}$ and $\gamma_{\mathrm{R}}$ are elliptic elements conjugate in $\widetilde{G}$.

An element $\gamma$ of $(\widetilde{G} \times \widetilde{G}) / \mathcal{Z}$ is synchronized if it is represented by a synchronized element of $\widetilde{G} \times \widetilde{G}$.

Lemma 4.3. ([7, Lemma 9.6]). An isometry $\gamma$ is synchronized if and only if there is an affine domain $U$ in $\widetilde{\mathrm{AdS}}$ such that $\gamma^{n}(U) \cap U \neq \emptyset$ for every $n$ in $\mathbb{Z}$. 
Observe

Lemma 4.4. ([7, Lemma 5.6]). Every generic, closed, achronal subset $\Lambda$ of $\widehat{\operatorname{Ein}}_{\mathrm{n}}$ is contained in a de Sitter domain.

Hence

Corollary 4.5. Any isometry preserving a generic, closed, achronal subset of $\widehat{\operatorname{Ein}}_{2}$ is synchronized.

\subsection{Causal open subsets}

Let $\gamma=\left(\gamma_{\mathrm{L}}, \gamma_{\mathrm{R}}\right)=\left(\exp \left(X_{\mathrm{L}}\right), \exp \left(X_{\mathrm{R}}\right)\right)$ be a synchronised element of $\widetilde{G} \times \widetilde{G}$.

Definition 4.6. The standard causal subset of $\gamma$, denoted by $C(\gamma)$, is the set of points $x$ of $\widetilde{\mathrm{AdS}}$ for which $\gamma x$ is not causally related to $x$.

Observe that $C(\gamma)=C\left(\gamma^{-1}\right)$, and $C(\gamma)$ is $\gamma$-invariant. The inclusions $C\left(\gamma^{k}\right) \subset C(\gamma)$ follow.

Definition 4.7. The convex causal subset of $\gamma$, denoted by $C_{\infty}(\gamma)$, is the set of points of $\widehat{\mathrm{AdS}}$ admitting in their future no $\gamma$-iterates of themselves.

Clearly, $C_{\infty}(\gamma)$ is the decreasing intersection of all $C\left(\gamma^{n}\right)$ when $n$ describes all $\mathbb{Z}$. It is $\gamma$-invariant.

At first glance, it seems natural to consider $C_{\infty}(\gamma)$ as the preferred $\gamma$ invariant subset such that the quotient is causal, i.e., does not admit closed causal curves (see [8, p. 7]). Actually, there exists a bigger subset with the same property which, in some way, is a maximal open subset with this property. The construction goes as follows: $\gamma$ is the time 1 map of the flow $\gamma^{t}=\left(\exp \left(t X_{\mathrm{L}}\right), \exp \left(t X_{\mathrm{R}}\right)\right)$ induced by some Killing vector field $X_{\gamma}$ of $\widetilde{\mathrm{AdS}}$.

Definition 4.8. The absolute causal subset of $\gamma$, denoted by $D(\gamma)$, is the open subset of $\widetilde{\operatorname{AdS}}$ where $X_{\gamma}$ is space-like.

Lemma 4.9. The open domain $D(\gamma)$ is the union of all the $C\left(\gamma^{1 / n}\right)$.

Clearly, since $X_{\gamma}$ is light-like on the boundary of $D(\gamma)$,

Lemma 4.10. Let $U \subset \widetilde{\mathrm{AdS}}$ be a $\gamma^{t}$-invariant subset containing $D(\gamma)$. If the quotient of $U$ by $\gamma$ is causal, then $U=D(\gamma)$. 
Proposition 4.11. If $\gamma$ is not a pair $\left(\gamma_{\mathrm{L}}, \gamma_{\mathrm{R}}\right)$ of elliptic elements with irrational rotation angle, the quotient space of $D(\gamma)$ by $\gamma$ is a strongly causal spacetime.

Proof. Denote

$$
\begin{aligned}
R_{0} & =\left(\begin{array}{cc}
0 & 1 \\
-1 & 0
\end{array}\right), \\
\Delta & =\left(\begin{array}{cc}
1 & 0 \\
0 & -1
\end{array}\right), \\
H & =\left(\begin{array}{ll}
0 & 1 \\
0 & 0
\end{array}\right) .
\end{aligned}
$$

Up to conjugacy in $\widetilde{G} \times \widetilde{G}$, inversion of time-orientation and permutation of the left-right components, we have seven cases to consider:

(1) $\left(X_{\mathrm{L}}, X_{\mathrm{R}}\right)=\left(\lambda R_{0}, \lambda R_{0}\right)(\lambda>0)$,

(2) $\left(X_{\mathrm{L}}, X_{\mathrm{R}}\right)=(\lambda \Delta, 0)(\lambda>0)$,

(3) $\left(X_{\mathrm{L}}, X_{\mathrm{R}}\right)=(H, 0)$,

(4) $\left(X_{\mathrm{L}}, X_{\mathrm{R}}\right)=(H,-H)$,

(5) $\left(X_{\mathrm{L}}, X_{\mathrm{R}}\right)=(H, H)$,

(6) $\left(X_{\mathrm{L}}, X_{\mathrm{R}}\right)=(\lambda \Delta, \mu \Delta)(0<\lambda \leq \mu)$,

(7) $\left(X_{\mathrm{L}}, X_{\mathrm{R}}\right)=(\lambda \Delta, H)(\lambda>0)$.

According to Lemmas 2.1, 2.3, the proposition is proved as soon as we check in every case that every connected component of $D(\gamma)$ is simply connected (when not empty).

For every $\tilde{g}$ in $\widetilde{\mathrm{AdS}} \approx \widetilde{G}$, the norm of $X_{\gamma}(\tilde{g})$ is $-\operatorname{det}$ of $X_{\mathrm{L}}-g X_{\mathrm{R}} g^{-1}=$ $X_{\mathrm{L}}-\operatorname{Ad}(g) X_{\mathrm{R}}$ (where $\left.g=p(\tilde{g})\right)$. It follows easily that in the cases (3) and $(4), D(\gamma)$ is actually empty.

Case (1): conjugacy by an elliptic element. In this case $D(\gamma)$ is $\widetilde{G} \backslash \mathbf{R}$, where $\mathbf{R}=\left\{\exp \left(t R_{0}\right)\right\}$, i.e., the complement of the set of fixed points of $\gamma$. The quotient of $D(\gamma)$ by the flow $\gamma^{t}$ is simply connected: apply Remark 2.2.

Case (2): translation by a hyperbolic element. In this case the action of $\gamma$ is free and properly discontinuous since it is an action by left translation. $D(\gamma)$ is the entire $\widetilde{G}$ : it is homeomorphic to $\mathbb{R}^{3}$, hence simply connected.

Case (5): conjugacy by a parabolic element. In this case $D(\gamma)$ is $p^{-1}(U)$, where $U \subset \mathrm{SL}(2, \mathbb{R})$ is the set of matrices:

$$
\left(\begin{array}{ll}
a & b \\
c & d
\end{array}\right) \quad a d-b c=1, c \neq 0 .
$$


For $g$ in $U$ the iterate $\exp (n H) g \exp (-n H)$ is the matrix

$$
\left(\begin{array}{cc}
a+n c & -n^{2} c+n(d-a)+b \\
c & -n c+d
\end{array}\right)
$$

Since $c \neq 0$ it follows easily that the action on $D(\gamma)$ is free and properly discontinuous. Every connected component of $D(\gamma)$ is simply connected.

Case (6): the hyperbolic-hyperbolic case. A straightforward calculus shows that in this case $\tilde{g}$ belongs to $D(\gamma)$ if and only if $b c<\frac{(\lambda-\mu)^{2}}{4 \lambda \mu}$, where $g=p(\tilde{g})$ is

$$
g=\left(\begin{array}{ll}
a & b \\
c & d
\end{array}\right) \quad a d-b c=1
$$

The projection in $G$ of the $\gamma^{n}$-iterate of $\tilde{g}$ is

$$
\left(\begin{array}{cc}
a \exp (n(\lambda-\mu)) & b \exp (n(\lambda+\mu)) \\
c \exp (-n(\lambda+\mu)) & d \exp (n(\mu-\lambda))
\end{array}\right) .
$$

If $\lambda \neq \mu$ then the action of $\gamma$ on the entire $\widetilde{\mathrm{AdS}}$ is free and properly discontinuous (see for example [23]). The strong causality of the action on $D(\gamma)$ is once more a corollary of Lemmas 2.1 and 2.3. When $\lambda=\mu$, the projection of $D(\gamma)$ is $\{b c<0\}$ : it is easy to see that the action on it is free and properly discontinuous due to Lemmas $2.1,2.3$, and we observe that $D(\gamma)$ is simply connected.

Case (7): the hyperbolic-parabolic case. This last case is completely similar to the previous one. The action of $\gamma$ on $\widetilde{\mathrm{AdS}}$ is free and properly discontinuous (see [23]), and $D(\gamma)$ is defined by

$$
-2 a c<\lambda .
$$

Details are left to the reader.

Remark 4.12. In case (6), if $\lambda \neq \mu$, the domain $D(\gamma)$ is not contained in an affine domain. This is an union of elementary domain of invisibility $\{b c<0\}$, connected by domains $\left\{0 \leq b c<\frac{(\lambda-\mu)^{2}}{4 \lambda \mu}\right\}$. The reader can find complementary descriptions in [11] or [5]. In the terminology of these papers, $\{b c<0\}$ is the union of regions of types I and II, and $\left\{0 \leq b c<\frac{(\lambda-\mu)^{2}}{4 \lambda \mu}\right\}$ are the regions of type III: the "inner regions". Compare, in particular, our proof of Proposition 4.11 with $[5, \S 3.2 .5]$. 
Remark 4.13. The region $D(\gamma)$ in case (7) is particularly difficult to draw. The best way to catch a picture of it is to consider this case as a limit of case (6): for every $\epsilon>0$ define $\gamma_{\epsilon}=\left(\gamma_{\mathrm{L}}, \gamma_{\mathrm{R}}^{\epsilon}\right)$, where

$$
\gamma_{\mathrm{R}}^{\epsilon}=\left(\begin{array}{cc}
\exp (\epsilon) & \frac{\sinh (\epsilon)}{\epsilon} \\
0 & \exp (-\epsilon)
\end{array}\right) \text {. }
$$

$\gamma_{\epsilon}$ is the exponential of $(\lambda \Delta, \epsilon \Delta+H)$. At the limit $\epsilon \rightarrow 0, \gamma_{\epsilon}$ tends to $\gamma$. Then $D(\gamma)$ is the limit of the domains $D\left(\gamma_{\epsilon}\right)$. Recall also Section 3.2.

Remark 4.14. When $\gamma_{\mathrm{L}}=\gamma_{\mathrm{R}}$ are elliptic elements with rational angle, the quotient of $\widehat{\mathrm{AdS}}$ by $\gamma$ is a singular spacetime with orbifold type. More precisely, the time-like line of $\gamma$ fixed points induces in the quotient a singular line which can be considered as the trajectory of a massive particle.

This point of view can be extended to the irrational angle case without difficulty, but we do not want to enter this discussion here. See for example $[12,18]$.

\section{Actions on invisible domains from elementary achronal subsets}

According to [7, Theorem 10.1],

Theorem 5.1. Let $\widetilde{\Lambda}$ be a non-elementary generic achronal subset, preserved by a torsion-free discrete group $\Gamma \subset \mathrm{SO}_{0}(2,2)$. Then the action of $\Gamma$ on $\Omega(\widetilde{\Lambda})$ and $E(\widetilde{\Lambda})$ is free, properly discontinuous, and the quotient spacetime $M_{\widetilde{\Lambda}}(\Gamma)$ is strongly causal.

This statement does not hold when $\widetilde{\Lambda}$ is elementary. The philosophy which should be retained is that in the elementary cases the invariant achronal subset $\widetilde{\Lambda}$, even if $\Gamma$-invariant is not sufficient to reveal the causal properties of $\Gamma$ : some points are missing (see Section 7 ).

\subsection{The extreme case}

Assume that $\widetilde{\Lambda}$ is extreme, i.e., is a light-like segment $[x, y]$. We can assume that the light-like geodesic $l$ containing $[x, y]$ is a leaf of the left foliation, i.e., an element of $\mathbb{R} P_{\mathrm{L}}^{1}$. Let $l_{x}, l_{y}$ be the right leaves, i.e., the elements of $\mathbb{R} P_{\mathrm{R}}^{1}$, containing respectively $x, y$. Then, $l$ is a fixed point of (the projection in $G$ ) of the left component $\gamma_{\mathrm{L}}$ of every element of $\Gamma$, and $l_{x}, l_{y}$ are fixed 
points of $\gamma_{\mathrm{R}}$. It follows easily that $\gamma$ is synchronized, that the right component $\gamma_{\mathrm{R}}$ is trivial or hyperbolic, and that the left component is non-elliptic (maybe trivial). In other words, with the notation involved in the proof of Proposition 4.11, $\gamma_{\mathrm{L}}=\exp (\lambda \Delta+\eta H)$ and $\gamma_{\mathrm{R}}=\exp (\mu \Delta)$.

The first commutator group $[\Gamma, \Gamma]$ is a group of left translations. Since $\Gamma$ is discrete, the same is true for $[\Gamma, \Gamma]$. Assume that $[\Gamma, \Gamma]$ is not trivial. Then it is a cyclic group $\operatorname{Aff}(\mathbb{R})$ of affine transformations of the line. A homothety of $\mathbb{R}$ cannot be a commutator of elements of $\operatorname{Aff}(\mathbb{R})$. Hence, in the last case above, $[\Gamma, \Gamma] \subset \widetilde{G}_{\mathrm{L}}$ is a cyclic group of parabolic elements preserved by conjugacies by left components of elements of $\Gamma$. It follows that these left components are necessarily parabolic, i.e., translations of $\mathbb{R}$. Hence, left components of elements of $[\Gamma, \Gamma]$ are trivial, which is a contradiction.

Therefore, $\Gamma$ is an abelian discrete subgroup of $A_{\text {hyp }}, A_{\text {ext }}$, where $A_{\text {hyp }}=$ $\{(\exp (\lambda \Delta), \exp (\mu \Delta))(\lambda, \mu \in \mathbb{R})\}$, and $A_{\text {ext }}=\{(\exp (\lambda H), \exp (\mu \Delta))(\lambda, \mu \in \mathbb{R})\}$.

\subsubsection{The mixed case $\Gamma \subset A_{\text {ext }}$}

In this case the action is free since a parabolic element can be conjugate in $\widetilde{G}$ to a hyperbolic one only if they are both trivial.

Claim: the action of $\Gamma$ on $E(\widetilde{\Lambda})$ is properly discontinuous.

Let us prove now the properness: assume by contradiction the existence of a compact $K$ in $E(\widetilde{\Lambda})$ and a sequence $\gamma_{n}=\left(\exp \left(\lambda_{n} H\right), \exp \left(\mu_{n} \Delta\right)\right)$ of elements of $\Gamma$ such that every $\gamma_{n} K \cap K$ is not empty. Let $\|$ be the operator norm on $\operatorname{gl}(E)$. Up to a subsequence, $\gamma_{n} /\left\|\gamma_{n}\right\|$ converges to an element $\bar{\gamma}$ of the unit ball of $\operatorname{gl}(E)$. Since $\Gamma$ is discrete, and since all the $\gamma_{n}$ have determinant one, the norms $\left\|\gamma_{n}\right\|$ tend to $+\infty$.

If the $\lambda_{n}$ are unbounded, up to a subsequence, we can assume that they tend to $+\infty$. Then the kernel of $\bar{\gamma}$ is a hyperplane and its image is a line. More precisely, the image is the line spanned by one of the extremities of $[x, y]$, let us say $x$; and the kernel is the $Q$-orthogonal $y^{\perp}$. The compact $K$ is disjoint from $y^{\perp}$ : it follows that in $P(E) \backslash y^{\perp}$, the sequence $\gamma_{n}$ converges uniformly on $K$ towards the constant map $x$. This is a contradiction, since $x$ does not belong to $K$.

If the $\lambda_{n}$ are bounded, the image and the kernel of $\bar{\gamma}$ are $Q$-isotropic 2planes (one of them is the 2-plane spanned by $x$ and $y$ ): their projection in $S(E)$ is disjoint from $\mathbb{A D S}$. But the iterates $\gamma_{n} K$ accumulate on the projection of the image of $\bar{\gamma}$ : we obtain a contradiction as above. The claim is proved. 
Moreover, according to Proposition 4.11, case (7), this action is strongly causal except if some right component $\exp (\mu \Delta)$ is trivial (case (3) of proposition 4.11): in this last case, the quotient is foliated by closed light-like geodesics, which are orbits of some 1-parameter subgroup of $A_{\text {ext }}$.

\subsubsection{The hyperbolic case $\Gamma \subset A_{\text {hyp }}$}

There is a particular situation: the subcase $\Gamma \subset \widetilde{G}_{\mathrm{L}}$. Then $\Gamma$ is cyclic. It follows from Proposition 4.11, case (2), that the action on $E(\widetilde{\Lambda})$ is free, properly discontinuous, and strongly causal. The same conclusion holds if $\Gamma \subset \widetilde{G}_{\mathrm{R}}$.

Hence, assume that $\Gamma$ is not contained in $\widetilde{G}_{\mathrm{R}}$ or $\widetilde{G}_{\mathrm{L}}$. The group $A_{\text {hyp }}$ admits four fixed points in $\overline{\operatorname{Ein}}_{2}$, including the projections of $x, y$. We can then define two additional $A_{\text {hyp }}$-fixed points $x^{\prime}, y^{\prime}$ uniquely defined by the requirement that $\left\{x, x^{\prime}\right\}$ and $\left\{y, y^{\prime}\right\}$ are strictly achronal.

Many subcases appear, with different behavior. For example, the action of $\Gamma$ on $E(\widetilde{\Lambda})$ may be free and properly discontinuous (for example, if $\Gamma$ is cyclic, spanned by an element for which $\lambda>\mu$ ). But the action may also be non-proper (the cyclic case, with $\lambda=\mu$ ). Anyway, this action is never causal. Indeed, if $\gamma$ is an element of $\Gamma \backslash\left(\widetilde{G}_{\mathrm{L}} \cup \widetilde{G}_{\mathrm{R}}\right), E(\widetilde{\Lambda})$ is $\gamma^{t}$-invariant, but is not contained in the absolute causal domain $D(\gamma)$. Then, the $\gamma^{t}$-orbit of a point $x$ in $E(\widetilde{\Lambda}) \backslash D(\gamma)$ is a time-like curve containing $x$ and $\gamma x$.

\subsection{The splitting case}

We consider the splitting case $\widetilde{\Lambda}=\{x, y\}$, with $x, y$ not causally related. Then the leaves of $\widehat{\mathcal{G}}_{\mathrm{R}}$ through $x, y$ are two distinct fixed points in $\mathbb{R} P_{\mathrm{R}}^{1}$. The right component of any element of $\Gamma$ is therefore trivial or hyperbolic. A similar argument shows that the left components are trivial or hyperbolic. Hence, in the notation of the previous section, we have $\Gamma \subset A_{\text {hyp }}$.

Observe that the segment $] x, y[$ is contained in $E(\widetilde{\Lambda})$. Hence, if $\Gamma$ is not cyclic, its action on $E(\widetilde{\Lambda})$ cannot be properly discontinuous.

Assume that $\Gamma$ is cyclic, spanned by $\gamma=(\exp (\lambda \Delta), \exp (\mu \Delta))$. If $\lambda$ or $\mu$ is zero the action is free, properly discontinuous, and causal.

If $\lambda$ and $\mu$ are both non-zero, it follows from case (6) of Proposition 4.11 that the action of $\Gamma$ on $E(\widetilde{\Lambda})$ is free, properly discontinuous, and strongly causal if and only if $x, y$ are attractive or repulsive fixed points of $\gamma$. 


\subsection{The conical case}

We assume here that $\widetilde{\Lambda}$ is conical, i.e., the union of two non-trivial lightlike segments $I_{1}=\left[x_{1}, x\right]$ and $I_{2}=\left[x, x_{2}\right]$. Then $\left\{x_{1}, x_{2}\right\}$ is strictly achronal, $E(\widetilde{\Lambda})$ is contained in $E\left(x_{1}, x_{2}\right)$, and $\Gamma$ preserves $E\left(x_{1}, x_{2}\right)$. As in the previous section we still have $\Gamma \subset A_{\text {hyp }}$.

Recall the description of $E(\widetilde{\Lambda})$ (Section 3.3): it is the union of the outer region $P_{2} \cap F_{2}$, the intermediate region $F_{2} \cap F_{1}$, and their common horizon boundary. It follows that $E(\widetilde{\Lambda})$ is $A_{\text {hyp }}$-invariant, and that all the $A_{\mathrm{hyp}^{-}}$ orbits inside $E(\widetilde{\Lambda})$ are 2-dimensional. Hence, the action of $A_{\text {hyp }}$ on $E(\widetilde{\Lambda})$ is free and properly discontinuous: the same is true for the action of $\Gamma$.

If $\Gamma$ is contained in $\widetilde{G}_{\mathrm{L}}$ or $\widetilde{G}_{\mathrm{R}}$, then its action on $E(\widetilde{\Lambda})$ is strongly causal. If not, the statement in the previous case still holds: the action of $\Gamma$ on $E(\widetilde{\Lambda})$ is free, properly discontinuous and strongly causal if and only if $\Gamma$ is cyclic and $x, y$ are attractive or repulsive fixed points of every non-trivial element of $\Gamma$.

\section{Existence of invariant achronal subsets}

Recall the following definition ([7, Definition 10.6]):

Definition 6.1. Let $\rho_{\mathrm{L}}: \Gamma \rightarrow G$ and $\rho_{\mathrm{R}}: \Gamma \rightarrow G$ be two morphisms. The representation $\rho=\left(\rho_{\mathrm{L}}, \rho_{\mathrm{R}}\right)$ is admissible if and only if it is faithful, has discrete image and lifts to some representation $\tilde{\rho}: \Gamma \rightarrow(\widetilde{G} \times \widetilde{G})_{/ \mathcal{Z}}$ preserving a generic closed achronal subset of $\widehat{\operatorname{Ein}}_{2}$ containing at least two points.

A $\rho$-admissible closed subset for an admissible representation $\rho$ is the projection in $\overline{\operatorname{Ein}}_{2}$ of $\tilde{\rho}$-invariant generic closed achronal subset of $\widehat{\operatorname{Ein}}_{2}$ containing at least two points.

In [7], we claimed (Theorem 10.7)

Theorem 6.2. Let $\Gamma$ be a torsion-free group and $\rho: \Gamma \rightarrow G \times G$ a faithful representation. Then $\rho$ is admissible if and only if one of the following occurs:

(1) The abelian case: $\rho(\Gamma)$ is a discrete subgroup of $A_{\mathrm{hyp}}, A_{\mathrm{ext}}$ or $A_{\mathrm{par}}$, where (see the notation in Section 5.1 where the first two groups are already defined) $A_{\text {hyp }}=\{(\exp (\lambda \Delta), \exp (\mu \Delta)) / \lambda, \mu \in \mathbb{R}\}, A_{\text {ext }}=\{(\exp$ $(\lambda \Delta), \exp (\eta H)) / \lambda, \eta \in \mathbb{R}\}$, and $A_{\text {par }}=\{(\exp (\lambda H), \exp (\lambda H)) / \lambda \in \mathbb{R}\}$. 
(2) The non-abelian case: The left and right morphisms $\rho_{\mathrm{L}}, \rho_{\mathrm{R}}$ are faithful with discrete image and the marked surfaces $\Sigma_{\mathrm{L}}=\rho_{\mathrm{L}}(\Gamma) \backslash \mathbb{H}^{2}, \Sigma_{\mathrm{R}}=$ $\rho_{\mathrm{R}}(\Gamma) \backslash \mathbb{H}^{2}$ are homeomorphic, i.e., there is a $\Gamma$-equivariant homeomorphism $f: \mathbb{H}^{2} \rightarrow \mathbb{H}^{2}$ satisfying

$$
\forall \gamma \in \Gamma, \quad f \circ \rho_{\mathrm{L}}(\gamma)=\rho_{\mathrm{R}}(\gamma) \circ f .
$$

Remark 6.3. Parabolic $\rho_{\mathrm{L}}(\gamma)$ correspond to punctures in $\Sigma_{\mathrm{L}}$. Hence, if $\gamma$ is a homotopy class corresponding to a loop which is not homotopic to an isolated end of $\Sigma_{R}, \rho_{\mathrm{L}}(\gamma)$ is necessarily hyperbolic.

Actually, we only proved in [7] the non-abelian case. Here, we justify the abelian case.

When $\Gamma$ is cyclic, a case-by-case study is needed, but which follows almost immediately from the study in the proof of Proposition 4.11. The situation can be summarized as follows:

Proposition 6.4. When $\Gamma$ is cyclic, then the representation $\rho$ is admissible if and only if either the left or right component of $\rho(\gamma)$ is hyperbolic and the other component non-elliptic, or if $\rho_{\mathrm{L}}(\gamma), \rho_{\mathrm{R}}(\gamma)$ are parabolic elements conjugate one to the other in $G$.

Assume now that $\Gamma$ is abelian but not cyclic. It follows from the cyclic case that $\rho$ is admissible if and only if $\rho(\Gamma)$ is contained in (a conjugate of $G$ of) $A_{\text {hyp }}, A_{\text {ext }}$, or $A_{\text {par }}$. The validity of Theorem 6.2 in the abelian case follows.

\section{Minimal invariant achronal subsets}

Let $\rho: \Gamma \rightarrow G \times G$ be an admissible representation.

Definition 7.1. $\bar{\Lambda}(\rho)$ is the closure of the set of attractive fixed points in $P(E)$.

Since attractive fixed points in $P(E)$ of elements of $G$ belong to $\overline{\operatorname{Ein}}_{2}$, $\bar{\Lambda}(\rho)$ is contained in $\overline{\operatorname{Ein}}_{2}$. According to $[7, \S 10.5]$ :

Theorem 7.2. Let $\Gamma$ be a non-abelian torsion-free group, and $\rho: \Gamma \rightarrow G \times$ $G$. Then every $\rho(\Gamma)$-invariant closed subset of $P(E)$ contains $\bar{\Lambda}(\rho)$.

Corollary 7.3. Let $(\Gamma, \rho)$ be a pair satisfying the hypothesis of Theorem 7.2. Then, $\bar{\Lambda}(\rho)$ is a $\rho(\Gamma)$-invariant generic non-elementary achronal subset of $\overline{\operatorname{Ein}}_{2}$. Furthermore, for every $\rho(\Gamma)$-invariant, closed, achronal subset $\Lambda$ 
in $\operatorname{Ein}_{2}$, the invisibility domain $E(\Lambda)$ projects injectively in $\overline{\mathbb{A D S}}$ inside $E(\bar{\Lambda}(\rho))$.

Remark 7.4. We will need the following remark: still assuming that $\Gamma$ is not abelian, the minimal closed achronal subset $\bar{\Lambda}(\rho)$ is the projection in $P(E)$ of $\Lambda(\rho) \cup-\Lambda(\rho)$, where $\Lambda(\rho)$ is a closed achronal subset of $\operatorname{Ein}_{2}$ and $-\Lambda(\rho)$ is the image of $\Lambda(\rho)$ by the antipody in $S(E)$. The action of $\rho(\Gamma)$ on $\Lambda(\rho)$ is minimal, hence $\Lambda(\rho)$ and $-\Lambda(\rho)$ are exactly the minimal components of the action of $\rho(\Gamma)$ on $S(E)$ (see [7, Lemma 10.21]).

When $\Gamma$ is abelian, Theorem 7.2 and Corollary 7.3 do not hold. However, in this case, still assuming that $\rho: \Gamma \rightarrow G$ is admissible:

- The extreme case: Let $l$ be the unique $A_{\text {ext }}$-invariant left leaf, and $r_{1}, r_{2}$ be the two $A_{\text {ext }}$ right-invariant right leaves. Let $\bar{\Lambda}_{\text {ext }}$ be the set of $A_{\text {ext }}-$ fixed points: $\bar{\Lambda}_{\text {ext }}=\left\{l \cap r_{1}, l \cap r_{2}\right\}$. It is easy to show that if $\rho(\Gamma) \subset$ $A_{\text {ext }}$, any generic $\rho(\Gamma)$-invariant, closed, achronal subset containing at least two points must contain $\bar{\Lambda}_{\text {ext }}$. This formulation is an extension - more accurately, a limit case - of Corollary 7.3, even if elements of $\rho(\Gamma)$ do not admit attractive fixed points in $P(E)$.

- The parabolic case: if $\rho(\Gamma) \subset A_{\text {par }}$, any $\rho(\Gamma)$-invariant closed subset contains the unique fixed point of $A_{\mathrm{par}}$. Hence, for any $\rho(\Gamma)$-invariant, closed, achronal subset $\Lambda$, we have $E(\Lambda) \subset D(\rho(\Gamma))$. This is in some way a limit case of the previous one.

- The hyperbolic-hyperbolic case: the group $A_{\text {hyp }}$ admits four fixed points in $P(E)$. If $\rho(\Gamma)$ is a lattice of $A_{\text {hyp }}$, it is easy to see that every $A_{\text {hyp }^{-}}$ fixed point is an attractive fixed point in $P(E)$ of some $\rho(\gamma)$. Anyway, Corollary 7.3 is false in this situation. There is however a convenient statement: any non-elementary, $\rho(\Gamma)$-invariant, generic, closed, achronal subset of $P(E)$ must contain the four fixed points of $A_{\text {hyp }}$.

\section{Coincidence of standard and absolute causal domains}

In Section 4.2, we have associated to a synchronized element $\gamma$ of $\widetilde{G} \times \widetilde{G}$ two open domains:

- the convex causal domain $C_{\infty}(\gamma)$,

- the absolute causal subset $D(\gamma)$.

In all cases, we have $C_{\infty}(\gamma) \subset D(\gamma)$. Actually, the identity $C_{\infty}(\gamma)=D(\gamma)$ holds if and only if $\gamma$ is a pair $\left(\gamma_{\mathrm{L}}, \gamma_{\mathrm{R}}\right)$, where $\gamma_{\mathrm{L}}, \gamma_{\mathrm{R}}$ are conjugate in $\widetilde{G}$. 
These definitions easily extend to (lifted) admissible representations $\widetilde{\rho}$ : $\Gamma \rightarrow \widetilde{G} \times \widetilde{G}$ :

Definition 8.1. The convex causal domain $C_{\infty}(\widetilde{\rho})$ is the interior of the intersection $\bigcap_{\gamma \in \Gamma} C_{\infty}(\widetilde{\rho}(\gamma))$. The absolute causal domain $D(\widetilde{\rho})$ is the interior of the intersection $\bigcap_{\gamma \in \Gamma} D(\widetilde{\rho}(\gamma))$.

The inclusions $C_{\infty}(\gamma) \subset D(\gamma)$ imply $C_{\infty}(\widetilde{\rho}) \subset D(\widetilde{\rho})$. Conversely,

Theorem 8.2. If $\Gamma$ is non-cyclic, then the convex causal domains and absolute causal domains coincide.

The rest of this section is devoted to the proof of Theorem 8.2.

\subsection{The flat case}

In the flat case, i.e., the case where $\widetilde{\rho}(\Gamma)$ preserves a point in $\widetilde{\mathrm{AdS}}$, the proof of Theorem 8.2 is obvious. Indeed, after conjugacy, we can assume in this case that the left and right representations $\rho_{\mathrm{L}}, \rho_{\mathrm{R}}$ coincide. Then, for every $\gamma$ in $\Gamma$, the identity $C_{\infty}(\rho(\gamma))=D(\gamma)$ holds.

\subsection{The abelian case}

If $\Gamma$ is abelian, since it is assumed non-cyclic, $\rho(\Gamma)$ is a lattice in $A_{\text {hyp }}$ or $A_{\text {ext }}$. We only consider the first case, the other can be obtained in a similar way (or as a limit case).

There are two morphisms $\alpha, \beta: \Gamma \rightarrow \mathbb{R}$ such that, for every $\gamma$ in $\Gamma$,

$\rho_{\mathrm{L}}(\gamma)=\left(\begin{array}{cc}\exp (\alpha(\gamma)) & 0 \\ 0 & \exp (-\alpha(\gamma))\end{array}\right), \quad \rho_{\mathrm{R}}(\gamma)=\left(\begin{array}{cc}\exp (\beta(\gamma)) & 0 \\ 0 & \exp (-\beta(\gamma))\end{array}\right)$

Recall that the projections in $\mathbb{A D S} \approx \mathrm{SL}(2, \mathbb{R})$ are

$$
C_{\infty}(\rho(\gamma))=\{b c<0\}, \quad D(\rho(\gamma))=\left\{b c<\sinh ^{2}(\alpha(\gamma)-\beta(\gamma))\right\}
$$

Theorem 8.2 follows then from the fact that, since $\rho(\Gamma)$ is a lattice in $A_{\text {hyp }},|\alpha(\gamma)-\beta(\gamma)|$ admits arbitrarily small value. 


\subsection{The proper case}

Assume that $\rho$ is strongly irreducible. The representations $\rho_{\mathrm{L}}$ and $\rho_{\mathrm{R}}$ are faithful, with discrete image, semi-conjugate to one another, but not conjugate in $G$, and $\Gamma$ is not abelian.

Let $\Lambda(\rho)$ be one of the two minimal closed subsets of $\mathcal{D} \subset S(E)$ such that the closure in $S(E)$ of the set of attractive fixed points is $\Lambda(\rho) \cup-\Lambda(\rho)$ (see Remark 7.4). It projects injectively on $\bar{\Lambda}(\rho)$, the closure in $P(E)$ of the set of attractive fixed points of elements of $\rho(\Gamma)$.

Let $E(\rho)$ be the invisible domain of $\Lambda(\rho)$ : this is the intersection between $\mathbb{A D S}$ and the intersection of all $\{x /\langle x \mid p\rangle<0\}$, where $p$ represents $\Lambda(\rho)$. Let $\bar{E}(\rho)$ be the projection of $E(\rho)$ in $\overline{\mathbb{A D S}}$. It can be defined in the following way:

$$
\bar{E}(\rho)=\{[x] \in \overline{\mathbb{A D S}} / \forall p, q \in \Lambda(\rho),\langle x \mid p\rangle\langle x \mid q\rangle>0\} .
$$

Indeed, although $\langle x \mid p\rangle,\langle x \mid q\rangle$ are not individually well defined for $[x]$ in $P(E)$, their product has a well-defined sign.

For any subset $J$ of $\bar{\Lambda}(\rho) \approx \Lambda(\rho)$, we can define $\bar{E}(J)$ as the interior of the set $\{[x] \in \overline{\mathbb{A D S}} / \forall[p],[q] \in J,\langle x \mid p\rangle\langle x \mid q\rangle>0\}$.

Lemma 8.3. If $J$ is $\rho(\Gamma)$-invariant and non-empty, then $\bar{E}(J)=\bar{E}(\rho)$.

Proof. The inclusion $\bar{E}(\rho) \subset \bar{E}(J)$ is obvious (observing that $\bar{E}(\rho)$ is open). The reverse inclusion follows from the fact that $\rho(\Gamma)$-invariant subsets of $\bar{\Lambda}(\rho)$ are dense, that $\bar{E}(J)$ is open if $J$ is closed, and that if $\bar{J}$ is the closure of $J, \bar{E}(\bar{J})=\bar{E}(J)$.

Corollary 8.4. Every connected component of $C_{\infty}(\widetilde{\rho})$ projects injectively in $P(E)$ on $\bar{E}(\rho)$.

Proof. Let $\gamma$ be a non-trivial element of $\Gamma$. We define $J(\gamma) \subset \overline{\mathcal{Q}}$ in the following way:

- If $\rho(\gamma)$ is hyperbolic-hyperbolic, $J(\gamma)=\left\{p^{+}(\gamma), p^{-}(\gamma)\right\}$, where $p^{+}(\gamma)$ is the attractive fixed point of $\rho(\gamma)$, and $p^{-}(\gamma)$ is the repulsive fixed point.

- If $\rho(\gamma)$ is hyperbolic-parabolic, $J(\gamma)=\{p(\gamma), q(\gamma)\}$, where $p(\gamma), q(\gamma)$ are the two $\rho(\gamma)$ fixed points,

- If $\rho(\gamma)$ is parabolic-parabolic, $J(\gamma)=\{p(\gamma)\}$, where $p(\gamma)$ is the unique fixed point. 
Then, the study in the proof of Proposition 4.11 shows that, in every case, every connected component of $C_{\infty}(\rho(\gamma))$ projects injectively on $\bar{C}_{\infty}(\rho(\gamma))=$ $\bar{E}(J(\gamma))$. On the other hand, if $\rho_{\mathrm{L}}(\gamma)$ (or $\left.\rho_{\mathrm{R}}(\gamma)\right)$ is parabolic, every closed subset of $\mathbb{R} P_{\mathrm{L}}^{1}$ (or $\mathbb{R} P_{\mathrm{R}}^{1}$ ) which is $\rho_{\mathrm{L}}(\gamma)$-invariant (or $\rho_{\mathrm{R}}(\gamma)$-invariant) contains the unique $\rho_{\mathrm{L}}(\gamma)$-fixed point (respectively the $\rho_{\mathrm{R}}(\gamma)$-fixed point). Hence, we have the inclusion $\bar{J}(\gamma) \subset \bar{\Lambda}(\rho)$. In other words, $\bar{C}_{\infty}(\rho(\gamma))$ is contained in $\bar{E}(\rho)$. Corollary 8.4 follows then from Lemma 8.3.

Corollary 8.5. Let $\gamma_{1}$ be an element of $\Gamma$ such that $\rho_{\mathrm{L}}\left(\gamma_{1}\right)$ and $\rho_{\mathrm{R}}\left(\gamma_{1}\right)$ are both hyperbolic. Then $C_{\infty}(\widetilde{\rho})=\bigcap_{\gamma \in \Gamma} C_{\infty}\left(\widetilde{\rho}\left(\gamma \gamma_{1} \gamma^{-1}\right)\right)$.

Proof. Corollary of Lemma 8.3 and Corollary 8.4, since $\bigcap_{\gamma \in \Gamma} \bar{C}_{\infty}\left(\rho\left(\gamma \gamma_{1} \gamma^{-1}\right)\right)$ is equal to $\bar{E}(J)$, where $J$ is the $\Gamma$-orbit of the attractive fixed points of $\rho\left(\gamma_{1}\right)$ and $\rho\left(\gamma_{1}^{-1}\right)$.

Proof of Theorem 8.2. According to Sections 8.1 and 8.2, we just have to consider the case where $\rho(\Gamma)$ is non-abelian and does not preserve a point in AdS. The surfaces $\Sigma_{\mathrm{L}}=\rho_{\mathrm{L}}(\Gamma) \backslash \mathbb{H}^{2}$ and $\Sigma_{\mathrm{R}}=\rho_{\mathrm{R}}(\Gamma) \backslash \mathbb{H}^{2}$ are homeomorphic: let $\Sigma$ be any surface homeomorphic to $\Sigma_{\mathrm{R}}, \Sigma_{\mathrm{L}}$.

Let $c_{1}$ be a closed loop in $\Sigma$ which is not freely homotopic to an isolated end of $\Sigma$. It represents a conjugacy class $\left[\gamma_{1}\right]$ in $\Gamma$. According to Remark 6.3, every $\rho_{\mathrm{L}, \mathrm{R}}\left(\gamma_{1}\right)$ is hyperbolic. After conjugacy, we can assume

$$
\rho_{\mathrm{L}}\left(\gamma_{1}\right)=\left(\begin{array}{cc}
\exp (\lambda) & 0 \\
0 & \exp (-\lambda)
\end{array}\right), \quad \rho_{\mathrm{R}}\left(\gamma_{1}\right)=\left(\begin{array}{cc}
\exp (\mu) & 0 \\
0 & \exp (-\mu)
\end{array}\right)
$$

with $\lambda \geq \mu>0$.

Since $\Gamma$ is non-abelian, the Euler characteristic of $\Sigma$ is negative. Hence, there is a closed loop $c_{2}$ in $\Sigma$, not freely homotopic to an end of $\Sigma$, and such that every loop freely homotopic to $c_{1}$ intersects every loop freely homotopic to $c_{2}$.

Let $\gamma_{2}$ be any element of $\Gamma$ corresponding to the free homotopy class of $c_{2}$. We express the coefficients of $\rho_{\mathrm{L}, \mathrm{R}}\left(\gamma_{2}\right)=\exp \left(A_{\mathrm{L}, \mathrm{R}}\right)$ :

$$
A_{\mathrm{L}}=\left(\begin{array}{cc}
\alpha_{\mathrm{L}} & \beta_{\mathrm{L}} \\
\nu_{\mathrm{L}} & -\alpha_{\mathrm{L}}
\end{array}\right), \quad A_{\mathrm{R}}=\left(\begin{array}{cc}
\alpha_{\mathrm{R}} & \beta_{\mathrm{R}} \\
\nu_{\mathrm{R}} & -\alpha_{\mathrm{R}}
\end{array}\right) .
$$

The fixed points in $\mathbb{R} P_{\mathrm{L}}^{1}$ of $\rho_{\mathrm{L}}\left(\gamma_{1}\right)$ are 0 and $\infty$. Hence, the connected components of the complement in $\mathbb{R} P_{\mathrm{L}}^{1}$ of these fixed points are ] $-\infty, 0[$ and $] 0,+\infty[$. 
The fixed points in $\mathbb{R} P_{\mathrm{L}}^{1}$ of $A_{\mathrm{L}}$ are $\alpha_{\mathrm{L}} \frac{1 \pm \sqrt{1+\beta_{\mathrm{L}} \nu_{\mathrm{L}} / \alpha_{\mathrm{L}}^{2}}}{\nu_{\mathrm{L}}}$. Replacing $\gamma_{2}$ by its inverse if necessary, and since the intersection between $c_{1}$ and $c_{2}$ is not trivial, we can assume that the attractive $\rho_{\mathrm{L}}\left(\gamma_{2}\right)$ fixed point belongs to ] $0,+\infty[$, and that the repulsive fixed point belongs to $]-\infty, 0[$. In other words, we can assume that the products $\beta_{\mathrm{L}} \nu_{\mathrm{L}}$ and $\alpha_{\mathrm{L}} \nu_{\mathrm{L}}$ are positive. Hence, after conjugacy by a diagonal matrix, we can assume $\nu_{\mathrm{L}}=\beta_{\mathrm{L}} \neq 0$.

Then, since $\rho_{\mathrm{L}}$ and $\rho_{\mathrm{R}}$ are semi-conjugate, the right components satisfy the same properties: we can assume $\beta_{\mathrm{R}}=\nu_{\mathrm{R}} \neq 0$.

Assume now by contradiction that the inclusion $C_{\infty}(\widetilde{\rho}) \subset D(\widetilde{\rho})$ is strict. Then, there is an element $\tilde{x}$ of $D(\widetilde{\rho})$ which is in the boundary of $C_{\infty}(\widetilde{\rho})$. Let $\widetilde{U}$ be an open neighborhood of $\tilde{x}$ in $D(\widetilde{\rho})$. Let $U$ be the projection of $\widetilde{U}$ in $P(E)$. Then, according to Corollary 8.5, there is an element $\gamma$ of $\Gamma$, a fixed point $x_{1}$ of $\rho\left(\gamma_{1}\right)$, and an element $p$ of $U$ such that $\left\langle\rho(\gamma) x_{1} \mid p\right\rangle=0$. After conjugacy of $\gamma_{1}$ by $\gamma$, we can assume that $\gamma$ is trivial. Moreover, we can also assume without loss of generality that $x_{1}$ is the attractive fixed point of $\rho\left(\gamma_{1}\right)$. Then, the equation $\left\langle x_{1} \mid p\right\rangle=0$ means $c=0$, where $p$ is expressed by the matrix

$$
g=\left(\begin{array}{ll}
a & b \\
c & d
\end{array}\right)
$$

Observe that we can also assume, after a slight modification of $p \approx g$ if necessary, that $b \neq 0$.

We consider the elements $\gamma_{n}=\gamma_{1}^{n} \gamma_{2} \gamma_{1}^{-n}$ of $\Gamma$. We have

$$
\begin{aligned}
& \rho_{\mathrm{L}}\left(\gamma_{n}\right)=\exp (n \lambda \Delta) \exp \left(A_{\mathrm{L}}\right) \exp (-n \lambda \Delta), \\
& \rho_{\mathrm{R}}\left(\gamma_{n}\right)=\exp (n \mu \Delta) \exp \left(A_{\mathrm{R}}\right) \exp (-n \mu \Delta) .
\end{aligned}
$$

Hence, the norm at $g$ of the Killing vector field generating $\gamma_{n}$ is the opposite of the determinant of $X_{n}$, with

$$
X_{n}=\exp (n \lambda \Delta) \exp \left(A_{\mathrm{L}}\right) \exp (-n \lambda \Delta) g-g \exp (n \mu \Delta) \exp \left(A_{\mathrm{R}}\right) \exp (-n \mu \Delta) .
$$

After computation, we see that $X_{n}$ is the matrix

$$
\left(\begin{array}{cc}
a\left(\alpha_{\mathrm{L}}-\alpha_{\mathrm{R}}\right)-b \beta_{\mathrm{R}} \exp (-2 n \mu) & b\left(\alpha_{\mathrm{L}}+\alpha_{\mathrm{R}}\right)+d \beta_{\mathrm{L}} \exp (2 n \lambda) \\
a \beta_{\mathrm{L}} \exp (-2 n \lambda)-d \beta_{\mathrm{R}} \exp (-2 n \mu) & b \beta_{\mathrm{L}} \exp (-2 n \lambda)-d\left(\alpha_{\mathrm{L}}-\alpha_{\mathrm{R}}\right)
\end{array}\right) .
$$

We distinguish two subcases: 
The case $\lambda>\mu$ : Observe that $b$ and $d$ are non-zero. If $\lambda>\mu$, the leading term of $-\operatorname{det}\left(X_{n}\right)$ for $n \rightarrow+\infty$ is

$$
-d^{2} \beta_{\mathrm{L}} \beta_{\mathrm{R}} \exp (2 n(\lambda-\mu)) .
$$

On the other hand, the leading term for $n \rightarrow-\infty$ is $b^{2} \beta_{\mathrm{L}} \beta_{\mathrm{R}} \exp (-2 n(\lambda+$ $\mu)$ ). But, since $g$ corresponds to an element of $D(\widetilde{\rho})$, all the $-\operatorname{det}\left(X_{n}\right)$ are positive. Hence, the product $\beta_{\mathrm{L}} \beta_{\mathrm{R}}$ must be positive and negative, which is a contradiction.

The case $\lambda=\mu$ : More precisely, the remaining case is the case where $\lambda=\mu$ for any choice of pairs $\gamma_{1}, \gamma_{2}$ as above, such that the corresponding homotopy classes have non-trivial intersection number. It is equivalent to the fact that $\operatorname{Tr}\left(\rho_{\mathrm{L}}\left(\gamma_{1}\right)\right)=\operatorname{Tr}\left(\rho_{\mathrm{R}}\left(\gamma_{1}\right)\right)$ for every $\gamma_{1}$ in $\Gamma$, which is not freely homotopic to a loop around an isolated end of $\Sigma$.

Select such a pair $\left(\gamma_{1}, \gamma_{2}\right)$ of $\Gamma$ satisfying the following additional property: the product $\gamma_{3}=\gamma_{1} \gamma_{2}$ is not freely homotopic to an isolated end of $\Sigma$ (we leave to the reader the proof of the fact that such a pair exists). Let $\Gamma_{1}$ be the group generated by $\gamma_{1}, \gamma_{2}$. Hence, we can assume the identity $\operatorname{Tr}\left(\rho_{\mathrm{L}}\left(\gamma_{i}\right)\right)=$ $\operatorname{Tr}\left(\rho_{\mathrm{R}}\left(\gamma_{i}\right)\right)$ for $i=1,2,3$. By Fricke-Klein Theorem [17, 19] these equalities imply that the restrictions of $\rho_{\mathrm{L}}$ and $\rho_{\mathrm{R}}$ to $\Gamma_{1}$ are representations conjugated in $\mathrm{SL}(2, \mathbb{R})$.

Therefore, after conjugacy, we can assume: $\rho_{\mathrm{L}}\left(\gamma_{i}\right)=\rho_{\mathrm{R}}\left(\gamma_{i}\right)(i=1,2)$. In other words, $\lambda=\mu, \alpha_{\mathrm{L}}=\alpha_{\mathrm{R}}, \beta_{\mathrm{L}}=\beta_{\mathrm{R}}$. A straightforward computation shows that the leading term of $-\operatorname{det}\left(X_{n}\right)$ for $n \rightarrow+\infty$ is $\left(a \beta_{\mathrm{L}}-d \beta_{\mathrm{R}}\right)\left(d \beta_{\mathrm{L}}-\right.$ $\left.a \beta_{\mathrm{R}}\right)=-(a-d)^{2} \beta_{\mathrm{L}}^{2}$. We obtain a contradiction since this term should be non-negative, whereas $d=1 / a \neq a$.

\section{Conformal boundaries of strongly causal spacetimes}

As we have seen in the introduction, the notion of black-hole is related to the notion of conformal boundary.

Definition 9.1. (Compare with $[13, \S 4.2]$ ). An AdS-spacetime with boundary is a triple $(M, O, \mathcal{M})$, where $\mathcal{M}$ is a manifold with boundary $O$ and interior $M$ which is $\left(\mathrm{AdS}, \operatorname{Ein}_{2}\right)$-modeled, i.e.:

- there exists a morphism (the holonomy representation) $\rho=\left(\rho_{\mathrm{L}}, \rho_{\mathrm{R}}\right)$ : $\Gamma \rightarrow G \times G$, where $\Gamma$ is the fundamental group of $\mathcal{M}$;

- there exists a $\rho$-equivariant local homeomorphism (the developing map) $\mathcal{D}: \widetilde{\mathcal{M}} \rightarrow \overline{\operatorname{Ein}}_{3}$, where $\widetilde{\mathcal{M}}$ is the universal covering of $\bar{M}$; 
- the image by $\mathcal{D}$ of $\widetilde{M}$, the interior of $\widetilde{\mathcal{M}}$, is contained in $\overline{\mathbb{A D S}}$;

- the image by $\mathcal{D}$ of the boundary $\widetilde{O}$ of $\widetilde{\mathcal{M}}$ is contained in $\overline{\operatorname{Ein}}_{2}$.

Observe that if $(M, O, \mathcal{M})$ is an AdS-spacetime with boundary, $M$ inherits a well-defined AdS-structure, and $O$ an $\operatorname{Ein}_{2}$-structure.

Our aim is to attach to any AdS-spacetime $M$ a AdS-spacetime with boundary $(M, O, \mathcal{M})$. This procedure should be canonical.

Definition 9.2. Let $(M, O, \mathcal{M}),\left(M^{\prime}, O^{\prime}, \mathcal{M}^{\prime}\right)$ be two AdS-spacetimes with boundary. A morphism between them is a local homeomorphism from $\mathcal{M}$ into $\mathcal{M}^{\prime}$ inducing an AdS-morphism $M \rightarrow M^{\prime}$.

Such a morphism lifts to a map $\tilde{F}: \widetilde{\mathcal{M}} \rightarrow \widetilde{\mathcal{M}^{\prime}}$ such that $\mathcal{D}^{\prime} \circ \tilde{F}=g \circ \mathcal{D}$ for some isometry $g$ of $\overline{\mathbb{A D S}}$. In particular, it induces an $\operatorname{Ein}_{2}$-morphism $O \rightarrow$ $O^{\prime}$. Such a morphism if an isomorphism if it is moreover a homeomorphism.

Definition 9.3. An AdS-spacetime with boundary $(M, O, \mathcal{M})$ is an universal conformal completion of $M$ if for any AdS-spacetime with boundary $\left(M, O^{\prime}, \mathcal{M}^{\prime}\right)$ there exists an injective morphism $\left(M, O^{\prime}, \mathcal{M}^{\prime}\right) \rightarrow(M, O, \mathcal{M})$.

It should be clear to the reader that if an AdS-spacetime $M$ admits an universal conformal completion, then this completion is unique up to isomorphism. In this case, the boundary is denoted by $O_{M}$, and called the natural conformal boundary of $M$.

Frances proved that complete AdS spacetimes, i.e., quotients of the entire AdS by discrete torsion-free subgroups, admit an universal completion ([14, Theorem 1]). But our spacetimes are never complete, and we will see that some of them do not admit universal conformal completion as defined above (see Remark 9.9). However, we can prove the existence of such universal completions if we restrict to the strongly causal category:

Observe first that causal curves in an AdS-spacetime with boundary is a well-defined notion since they are well defined in $\overline{\mathbb{A D S}} \cup \overline{\operatorname{Ein}}_{2}$. We can therefore define the causality relation in such a manifold with boundary, and in particular the strong causality property (see Section 2).

Definition 9.4. An AdS-spacetime with boundary $(M, O, \mathcal{M})$ is an universal strongly causal conformal completion of $M$ if it is strongly causal, and for any strongly causal AdS-spacetime with boundary $\left(M, O^{\prime}, \mathcal{M}^{\prime}\right)$ there exists an injective morphism $\left(M, O^{\prime}, \mathcal{M}^{\prime}\right) \rightarrow(M, O, \mathcal{M})$.

Obviously, an AdS-spacetime can admit a conformal strongly causal completion only if it is already strongly causal. Conversely, 
Theorem 9.5. Every strongly causal AdS-spacetime admits an universal strongly causal conformal completion.

In the proof we will need the following lemmas, valid for any local homeomorphism $\varphi: X \rightarrow Y$ between manifolds (for proofs, see for example $[6, \S 2.1])$ :

Lemma 9.6 (Lemme des assiettes emboîtées). Let $U, U^{\prime}$ be two open domains in $X$ such that the restrictions of $\varphi$ on $U, U^{\prime}$ are injective. Assume that $U \cap U^{\prime}$ is not empty and that $\varphi\left(U^{\prime}\right)$ contains $\varphi(U)$. Then $U^{\prime}$ contains $U$.

Lemma 9.7 (Fermeture des assiettes). Assume that $\varphi$ is injective on some open domain $U$ in $X$, and that the image $V=\varphi(U)$ is locally connected in $Y$, i.e., that every point $y$ in the closure of $V$ admits arbitrarily small neighborhood $W$ such that $V \cap W$ is connected. Then, the restriction of $\varphi$ to the closure of $U$ in $X$ is injective.

Proof of Theorem 9.5. Step 1: the construction of the AdS-spacetime with conformal boundary $(\widetilde{M}, \widetilde{O}, \widetilde{\mathcal{M}})$. We need to start with a definition: an end in $\widetilde{M}$ is an open domain $U$ in $\widetilde{M}$ such that the restriction of $\mathcal{D}$ to $U$ is injective, with image an end $V$ in $\overline{\mathbb{A D S}}$ (see Definition 3.2, figure 2). The proof relies on the geometric understanding of ends, hence we insist on their description: an end is the intersection $F \cap P$, where $P$ is the past of an element $a$ of $\overline{\operatorname{Ein}}_{2}, F$ the future of an element $b$ of $\overline{\operatorname{Ein}}_{2}$, and such that $a, b$ are strictly causally in a de Sitter domain containing $V$. The interior in $\overline{\operatorname{Ein}}_{2}$ of the intersection between $\overline{\operatorname{Ein}}_{2}$ and the closure of $V$ is a diamond-shaped region, denoted by $\partial U$. The end itself is the intersection between $\overline{\mathbb{A D S}}$ and the convex hull in $P(E)$ of the boundary of $\partial U$.

Let $\bar{U}$ be the union $U \cap \partial U$. Observe that for any end $U$ the triple $(U, \partial U, \bar{U})$ is an AdS-spacetime with boundary. A marked end is a pair $(U, x)$, where $U$ is an end in $\widetilde{M}$ and $x$ an element of $\partial U \subset \overline{\operatorname{Ein}}_{2}$. Let $\mathcal{E}$ be the set of marked ends in $\widetilde{M}$. On $\mathcal{E}$, let $\sim$ be the equivalence relation identifying two marked ends $(U, x),\left(U^{\prime}, x\right)$ if there is a third marked end $\left(U^{\prime \prime}, x\right)$ with $U^{\prime \prime} \subset U \cap U^{\prime}$. Let $\Upsilon$ be the quotient space of $\sim$. Let $\Xi$ be the union $\Upsilon \cup \widetilde{M}$. For any end $U$, let $\partial \widehat{U} \subset \Upsilon$ be the set $\left\{\left[U^{\prime}, y\right] \in \Upsilon / U^{\prime} \subset U\right\}$, and let $\widehat{U}$ be the union in $\Xi$ of $\partial \widehat{U}$ with $U$. The $\partial \widehat{U}$ form the basis of a topology on $\Upsilon$, and the $\widehat{U}$ form, with the open subset of $\widetilde{M}$, the basis of a topology in $\Xi$. It should be clear to the reader that in the special case where $\widetilde{M}$ is the end $V$, all this process gives as final output topological spaces $\Upsilon$, $\Xi$ respectively homeomorphic to $\partial V, V \cup \partial V$, where $\partial V$ is the interior of the intersection between $\overline{\operatorname{Ein}}_{2}$ and the closure of $V$. 
The inclusion $\widetilde{M} \subset \Xi$ is clearly a homeomorphism onto its image, which is dense in $\Xi$. Similarly, for any marked end $(U, x)$, the open domain $\widehat{U}$ is a neighborhood of $[U, x]$ in $\Xi$, which is homeomorphic to $V \cup \partial V$, the conformal completion in $\overline{\operatorname{Ein}}_{3}$ of the end $V=\mathcal{D}(U)$ in $\overline{\mathbb{A D S}}$. It follows that $\Xi$ is a manifold with boundary, admitting as chards the chards of $\widetilde{M}$ and the open subsets $\widehat{U}$. Indeed, the only remaining point to check (with the second countability that we leave to the reader) is the Hausdorff property: let $x_{1}$, $x_{2}$ be two elements of $\Xi$ such that every neighborhood of $x_{1}$ intersects every neighborhood of $x_{2}$. Then, clearly, if $x_{1}$ belongs to $\widetilde{M}$, the same is true for $x_{2}$, and $x_{1}=x_{2}$. If not, $x_{1}$ and $x_{2}$ belong to $\Upsilon: x_{1}=\left[U_{1}, x_{1}^{\prime}\right], x_{2}=\left[U_{2}, x_{2}^{\prime}\right]$ with $\left(U_{i}, x_{i}\right) \in \mathcal{E}$. By hypothesis, the neighborhoods $\widehat{U}_{1}$ and $\widehat{U}_{2}$ must overlap. If $U_{1} \cap U_{2} \subset \widetilde{M}$ is empty, then some $\left[U_{3}, x_{3}\right]$ must belong to $\widehat{U}_{1} \cap \widehat{U}_{2}$. Then, points in $U_{3}$ correspond to points in $\widehat{U}_{3}$ which are in $U_{1} \cap U_{2}$, a contradiction. Hence, $U_{1} \cap U_{2}$ must intersect for any choice of the marked ends $\left(U_{i}, x_{i}\right)$. Fix one choice $\left(U_{i}^{0}, x_{i}^{0}\right)$ of these ends, and consider for every $i=1,2$ smaller ends $U_{i} \subset U_{i}^{0}$. More precisely, fix $U_{1}$, with $\left[U_{1}^{0}, x_{1}\right]=\left[U_{1}, x_{1}\right]$ and $U_{1} \subset U_{1}^{0}$. Then, if $U_{2}$ is sufficiently small, its image $\mathcal{D}\left(U_{2}\right)$, which intersects $\mathcal{D}\left(U_{1}\right)$, is contained in $\mathcal{D}\left(U_{1}^{0}\right)$. According to Lemma 9.6, $U_{2}$ is then contained in $U_{1}^{0}$. But, since $x_{1}, x_{2}$ are not separated one from the other, they must have the same image under $\mathcal{D}$. Applying Lemma 9.7, we obtain $x=y$, i.e., $\Xi$ is Hausdorff.

The combination of the developing map $\mathcal{D}: \widetilde{M} \rightarrow \overline{\mathbb{A D S}}$ with the inclusions $\bar{U} \subset \overline{\operatorname{Ein}}_{3}$ induces a well-defined continuous map $\mathcal{D}: \Xi \rightarrow \overline{\operatorname{Ein}}_{3}$. Moreover, the restriction of $\mathcal{D}$ to (the projection of) any closed end $\bar{U}$ is injective: $\mathcal{D}$ is a local homeomorphism.

Finally, the action of $\Gamma$ on $\widetilde{M}$ extends naturally on $\Xi$ : for any $\gamma$ in $\Gamma$, define $\gamma[x \in \bar{U}]$ as being $[\rho(\gamma) x \in \rho(\gamma) \bar{U}]$.

Observe that this action is continuous and preserves $\Upsilon=\Xi \backslash \widetilde{M}$. Moreover, the map $\mathcal{D}: \Xi \rightarrow \overline{\operatorname{Ein}}_{3}$ is equivariant for this action.

We can now define $\widetilde{\mathcal{M}}$ : this is the set of $\Gamma$-causally wandering points in $\Xi$, i.e., the set of elements $x$ of $\Xi$ admitting neighborhood $W$ such that for every non-trivial element $\gamma$ of $\Gamma$ no element of $W$ is causally related in $\Xi$ to an element of $\gamma W$. Observe that $\Xi$ is open, $\Gamma$-invariant, and that it contains $\widetilde{M}$ since the action of $\Gamma$ on $\widetilde{M}$ is strongly causal. $\widetilde{O}$ is the complement $\widetilde{\mathcal{M}} \backslash \widetilde{M}$ (hence, the set of $\Gamma$ non-causally wandering points).

Step 2: the action of $\Gamma$ on $\widetilde{\mathcal{M}}$ is free, proper, and strongly causal. Observe that the action is free, since the fixed points are not wandering. Assume that the action of $\Gamma$ on $\widetilde{\mathcal{M}}$ is not proper. 
Then, there are sequences $\left(x_{n}\right)_{(n \in \mathbb{N})},\left(y_{n}\right)_{(n \in \mathbb{N})}$ in $\widetilde{\mathcal{M}}$ and a sequence $\gamma_{n}$ in $\Gamma$ such that:

$$
\begin{aligned}
& -\gamma_{n} x_{n}=y_{n}, \widetilde{\mathcal{M}}, \\
& -x_{n} \rightarrow x \in \widetilde{\mathcal{M}}, \text { and } \\
& -y_{n} \rightarrow y \text { is not in the } \Gamma \text {-orbit of } x . \\
& -y \text { is }
\end{aligned}
$$

Let $\bar{x}=\mathcal{D}(x), \bar{y}=\mathcal{D}(y), \bar{x}_{n}=\mathcal{D}\left(x_{n}\right), \bar{y}_{n}=\mathcal{D}\left(y_{n}\right)$. We also decompose $g_{n}=\rho\left(\gamma_{n}\right)$ along their left and right components: $g_{n}=\left(g_{\mathrm{L}}^{n}, g_{\mathrm{R}}^{n}\right)$.

Since the action of $\Gamma$ on $\widetilde{M}$ is proper (it is the group of covering automorphisms), $x$ or $y$ must belong to $\widetilde{O}$; let us say $x$. Then $x$ belongs to a diamond shaped region $\partial U$, where $U$ is an end in $\widetilde{M}$. Since $x$ is $\Gamma$-wandering, we can choose $U$ so that $\gamma \bar{U} \cap \bar{U}=\emptyset$ for every non-trivial $\gamma$ in $\Gamma$. Moreover, since $\bar{U}$ is open in $\widetilde{\mathcal{M}}$, we can assume that all $x_{n}$ belong to $U$.

Define $U_{n}=\gamma_{n} U, \Delta=\partial U, \Delta_{n}=\gamma_{n} \Delta$, and $\bar{\Delta}_{n}=\mathcal{D}\left(\Delta_{n}\right), \bar{U}_{n}=\mathcal{D}\left(U_{n}\right)$. The image $\bar{\Delta}=\mathcal{D}(\Delta)$ is a diamond-shaped region. Since the $U_{n}$ are necessarily disjoint we can assume that none of them contains $y$.

Claim: $y$ belongs to $\widetilde{O}$.

Assume not. There is a small neighborhood $W$ of $y$ in $\widetilde{M}$ such that the restriction of $\mathcal{D}$ is injective with image a small ellipsoid $\bar{W}$ in $\overline{\mathbb{A D S}}$. For $n$ sufficiently big $y_{n}$ belongs to $W$, hence the intersection $\mathcal{I}_{n}=W \cap U_{n}$ is not empty. On the other hand, $\bar{U}_{n}$ is an end: it is a connected component of the intersection between $\overline{\mathbb{A D S}}$ and the complement of two hyperplanes in $P(E)$. It follows that the intersection $\overline{\mathcal{I}}_{n}$ between the ellipsoid $\bar{W}$ and $\bar{U}_{n}$ is convex: it is the trace in an ellipsoid of a half-space or a quarter of space. Moreover, the restriction of $\mathcal{D}$ to $\mathcal{I}_{n}$ and $W$ is injective. Hence, according to Lemma 9.6 , the image of $\mathcal{I}_{n}$ by $\mathcal{D}$ is the entire $\overline{\mathcal{I}}_{n}$. In other words, the screen $\bar{W}$ reflects faithfully how the ends $U_{n}$ intersect $W$. But it is geometrically clear that $\bar{U}_{n}$ cannot accumulate to $\bar{y}$ if they are disjoint one to the other: visualize by considering an ellipsoid $W^{\prime}$ in the Minkowski space conformally equivalent to $\bar{W}$; then, in this conformal chord, the $\bar{U}_{n}$ are intersections between the past of light-like plane and the future of another light-like plane. It leads to a contradiction: the claim is proved.

Replace the ellipsoid $W$ in the proof above by a neighborhood $\bar{U}^{\prime}$ with $U^{\prime} \in \mathcal{E}$ and such that $y$ belongs to $\partial U^{\prime}$. We can assume that all the $y_{n}$ belong to $U^{\prime}$. The argument above, based on Lemma 9.6, shows that the image by 
$\mathcal{D}$ of the intersection $U_{n} \cap U^{\prime}$ is also the entire $\bar{U}^{\prime} \cap \bar{U}_{n}$. Applying once more this lemma to the closure, we obtain that $\mathcal{D}$ projects faithfully the intersections between $\Delta^{\prime}$ and $\Delta_{n}$ over the entire $\bar{\Delta}_{n} \cap \bar{\Delta}^{\prime}$. But these intersections are even simpler to visualize than intersection between an ellipsoid and ends: indeed, through the identification $\overline{\mathbb{A D S}} \approx \mathbb{R} P_{\mathrm{L}}^{1} \times \mathbb{R} P_{\mathrm{R}}^{1}$, a diamond-shaped region corresponds to the product of two open intervals $I_{\mathrm{L}} \times I_{\mathrm{R}}$. Denote by $I_{\mathrm{L}}^{0}, I_{\mathrm{R}}^{0}$ the open intervals in the projective line such that $I_{\mathrm{L}}^{0} \times I_{\mathrm{R}}^{0}=\partial \bar{\Delta}^{\prime}$ : $\bar{y}$ corresponds to a pair $\left(y_{\mathrm{L}}, y_{\mathrm{R}}\right) \in I_{\mathrm{L}}^{0} \times I_{\mathrm{R}}^{0}$. For every integer $n$ let $I_{\mathrm{L}}^{n} \subset I_{\mathrm{L}}^{0}$ and $I_{\mathrm{L}}^{n} \subset I_{\mathrm{R}}^{0}$ be the intervals such that $\bar{\Delta}_{n} \cap \bar{\Delta}^{\prime}=I_{\mathrm{L}}^{n} \times I_{\mathrm{R}}^{n}$.

Assume that for some integers $n \neq m$ the intersection $I_{\mathrm{L}}^{n} \cap I_{\mathrm{L}}^{m}$ is not empty. Then there is light-like segment in $\bar{\Delta}^{\prime}$ with one extremity in $\bar{\Delta}_{n}$ and the other in $\bar{\Delta}_{m}$. It follows that there is a causal curve joining an element of $U_{n} \cap U^{\prime}$ to an element of $U_{m} \cap U^{\prime}$. Now, for the first time, we use the fact that $M$ is strongly causal, i.e., that the action of $\Gamma$ on $\widetilde{M}$ is strongly causal: it means that the open domain $U$ can be selected so that for every non-trivial element of $\Gamma$ no element of $\gamma U$ can be causally related to an element of $U$. Apply this remark to $\gamma_{n}^{-1} \gamma_{m}$ : we obtain a contradiction.

Hence, for every $n \neq m$ we have $I_{\mathrm{L}}^{n} \cap I_{\mathrm{L}}^{m}=\emptyset$. Similarly $I_{\mathrm{R}}^{n} \cap I_{\mathrm{R}}^{m}=\emptyset$. But since the $y_{n}$ converge to $y=\left(y_{\mathrm{L}}, y_{\mathrm{R}}\right)$ it follows that $\left(I_{\mathrm{L}}^{n}\right)_{(n \in \mathbb{N})}$ (respectively $\left.\left(I_{\mathrm{R}}^{n}\right)(n \in \mathbb{N})\right)$ is a decreasing sequence of intervals converging uniformly to $\left\{y_{\mathrm{L}}\right\}$ (respectively $\left\{y_{\mathrm{R}}\right\}$ ). Hence for big $n$ we have $U_{n} \subset U^{\prime}$. We obtain a contradiction since $U^{\prime}$ can be chosen so that $\gamma U^{\prime} \cap U^{\prime}=\emptyset$ for every non-trivial $\gamma$.

This final contradiction achieves the proof of step 2. Hence, the quotient $\mathcal{M}=\Gamma \backslash \widetilde{\mathcal{M}}$ is a manifold, with boundary $O=\Gamma \backslash \widetilde{O}$.

Step 3: $(M, O, \mathcal{M})$ is an universal conformal completion. Let $\left(M, O^{\prime}, \mathcal{M}^{\prime}\right)$ be another conformal completion of $M$. Let $\pi^{\prime}: \widetilde{\mathcal{M}}^{\prime} \rightarrow \mathcal{M}^{\prime}$ be the universal covering of $\mathcal{M}$. The interior of $\widetilde{\mathcal{M}}^{\prime}$ is simply connected, hence it can be identified with the universal covering of $M$. Hence $M, \mathcal{M}^{\prime}$ (and $\mathcal{M}$ ) have the same fundamental group $\Gamma$. Let $\mathcal{D}^{\prime}: \widetilde{\mathcal{M}}^{\prime} \rightarrow \overline{\operatorname{Ein}}_{3}$ and $\rho: \Gamma \rightarrow G \times G$ be the developing map and the holonomy representation of $(M, O, \mathcal{M})$. The developing map for $M$ is then the restriction to $\widetilde{M}$ of $\mathcal{D}^{\prime}$, and $\rho$ is the holonomy representation of the AdS-spacetime $M$.

The main observation is the following: every point $x$ in $\widetilde{O}^{\prime}$ admits a neighborhood $U^{\prime}$ in $\widetilde{\mathcal{M}}^{\prime}$ such that the restriction of $\mathcal{D}^{\prime}$ to $U^{\prime}$ is injective and the image $\mathcal{D}^{\prime}(U \cap \widetilde{M})$ is an end of $A d S$. Hence, $\left(U \cap \widetilde{M}, \mathcal{D}^{\prime}(x)\right)$ is a marked end of $\widetilde{M}$. It defines a map $\widetilde{O} \rightarrow \Upsilon$. With the identity map $\widetilde{M} \rightarrow \widetilde{M}$, and after composition in the quotient space, we obtain a map $\widetilde{F}: \widetilde{\mathcal{M}}^{\prime} \rightarrow \Xi$. The proof 
that $\widetilde{F}$ is a homeomorphism onto its image is straightforward and left to the reader.

The main point is to show that $\widetilde{F}$ takes value in the domain of $\Gamma$-wandering points $\widetilde{\mathcal{M}}$. Assume by contradiction the existence of a point $x$ in $\widetilde{\mathcal{M}}^{\prime}$ such that $x^{\prime}=\widetilde{F}(x)$ is not a $\Gamma$-wandering point. Since $\Gamma$ acts properly on $\widetilde{\mathcal{M}}^{\prime}$, there is a neighborhood $W$ of $x$ such that $\gamma W \cap W=\emptyset$ for every non-trivial $\gamma$. Moreover, since it is not $\Gamma$-wandering, $x^{\prime}$ does not belong to $\widetilde{M}$. Hence, $x$ belongs to $\widetilde{O}^{\prime}$ : we can choose $W$ so that $W \cap \widetilde{M}$ is an end of $\widetilde{M}$. Then, by hypothesis, there is a non-trivial $\gamma$ such that $\gamma \bar{W} \cap \bar{W}$ is not empty since $\bar{W}$ is a neighborhood of $x^{\prime}$ in $\widetilde{\mathcal{M}}$. But complete ends overlapping in $\widetilde{\Xi}$ must also overlap in the interior of $\widetilde{\Xi}$, i.e., in $\widetilde{M}$. Hence, this overlapping exists also in $\widetilde{\mathcal{M}}^{\prime}$, which is a contradiction.

Hence, $\widetilde{F}$ takes value in $\widetilde{\mathcal{M}}$. Since the construction is $\Gamma$-equivariant, $\widetilde{F}$ induces the required morphism $\left(M, \mathcal{M}^{\prime}, O^{\prime}\right) \rightarrow(M, O, \mathcal{M})$.

Remark 9.8. Consider the quotient of $\widetilde{\mathrm{AdS}}$ by a cyclic group $\Gamma$ generated by a hyperbolic left translation $\gamma=\left(\gamma_{\mathrm{L}}, i d\right)$. Then the set of fixed points of $\gamma$ is the union of two left light-like leaves $l_{1}, l_{2}$. The action of $\Gamma$ on $\widetilde{\mathrm{AdS}} \cup \widehat{\operatorname{Ein}}_{2} \backslash\left(l_{1} \cup l_{2}\right)$ is free and properly discontinuous, hence the quotient space is an universal conformal boundary for $\Gamma \backslash \widetilde{\mathrm{AdS}}$. But since every $\Gamma$ orbit of elements of $\widehat{\operatorname{Ein}}_{2}$ is contained in a right light-like leaf, it follows that the strongly causal conformal boundary of $\Gamma \backslash \widetilde{\operatorname{AdS}}$ is empty.

Remark 9.9. Restricting to the strongly causal category is essential to ensure the uniqueness of the maximal conformal extension. Indeed, let $\gamma=$ $(\exp (\lambda \Delta), \exp (\mu \Delta))$ be a hyperbolic-hyperbolic element of $\widetilde{G} \times \widetilde{G}$ with $\lambda \neq$ $\mu$. The action of $\gamma$ on $\widetilde{\mathrm{AdS}}$ is free and properly discontinuous. In $\overline{\operatorname{Ein}}_{2}$ there are two $\gamma$-invariant left leaves $l_{1}, l_{2}$, and two $\gamma$-invariant right leaves $r_{1}, r_{2}$.

The preimage $\tilde{l}_{i}, \tilde{r}_{i}$ in $\widehat{\operatorname{Ein}}_{2}$ of $l_{i}, r_{i}$ are light-like geodesics. It is easy to see that the action of $\Gamma$ on $\widehat{\operatorname{AdS}} \cup \widehat{\operatorname{Ein}}_{2} \backslash\left(l_{1} \cup l_{2}\right)$ is free and properly discontinuous, and the same is true for the action on $\widetilde{\operatorname{AdS}} \cup \widehat{\operatorname{Ein}}_{2} \backslash\left(r_{1} \cup r_{2}\right)$. Each of these extensions provides a conformal completion of $\Gamma \backslash \widetilde{\mathrm{AdS}}$. But it can be proved that each of this extensions is maximal, i.e., does not embed in a larger conformal completion of $\Gamma \backslash \widehat{\operatorname{AdS}}$. Hence $\Gamma \backslash \widehat{\operatorname{AdS}}$ does not admit a universal conformal completion.

A similar situation appears in the so-called "Taub - NUT" examples; see $[17,20]$. 


\section{BTZ black holes and multi-black-holes}

In this section, we consider various pairs $(\rho, \Lambda)$, where $\rho=\left(\rho_{\mathrm{L}}, \rho_{\mathrm{R}}\right): \Gamma \rightarrow$ $G \times G$ is an admissible representation and $\Lambda$ a $\rho$-admissible closed subset of $\overline{\operatorname{Ein}}_{2}$ (see Definition 6.1). We prove in the selected cases that the spacetimes $M_{\Lambda}(\Gamma)$ are AdS-spacetimes with black-holes in the sense of Definition 1.2.

Remark 10.1. We do not pretend to study all the possibilities: for example, for non-abelian $\Lambda$, we only consider the case $\Lambda=\Lambda(\Gamma)$, whereas the case $\Lambda \neq \Lambda(\Gamma)$ gives other examples of spacetimes with black-holes. Moreover, we do not consider the case where $\Gamma$ is trivial, which would lead, for the $\Lambda$ selected below, to AdS-spacetimes with black-hole too!

A justification for this omission is that all these spacetimes are not maximal for (too much) obvious reasons, because of the embedding $M_{\Lambda}(\Gamma) \subset$ $M_{\Lambda(\Gamma)}(\Gamma)$ - especially in the case $\Gamma=\mathrm{id}$, where the whole spacetime embeds in $\widehat{\mathrm{AdS}}$.

\subsection{The conical black-holes}

This is the case where $\Lambda$ is conical (see Section 3.3). More precisely, we have to consider the case where $\Lambda$ is a upper lower tent $\mathcal{T}_{x y}^{+}=[x, z] \cup[z, y]$ (if it is a lower tent, then the spacetime is full, but without a black-hole). The domain $E(\Lambda)$ has been described in Section 3.3. From this description (see also figure 2), it appears clearly that the conformal boundary $\widetilde{O}$ of $E(\Lambda)$ is the diamond-shaped region $\Delta_{2}=\Omega(\Lambda)$, and the completion of $E(\Lambda)$ is $\mathcal{M}(\Lambda)=E(\Lambda) \cup \Delta_{2}$. The region $F_{1} \cap F_{2}$ is the region invisible from $\widetilde{O}$. We can understand a part of the terminology reported in Remark 3.5: the outer region $F_{2} \cap P_{2}$ is the region visible to observers in $O$.

According to Section 5.3 the action of $\Gamma$ on $E(\Lambda)$ is free, properly discontinuous, and strongly causal if and only if $\Gamma$ is a cyclic group generated by an element $\gamma=\left(\gamma_{\mathrm{L}}, \gamma_{\mathrm{R}}\right)=(\exp (u \Delta), \exp (v \Delta))$. We can assume without loss of generality that $v \geq u \geq 0$. It appears then clearly that the action of $\Gamma$ on $\widetilde{O}=\Delta_{2}$ is free and proper. Moreover, this action is strongly causal if $u=0$. Hence, its quotient $O$ is the natural conformal boundary of $M_{\Lambda}(\Gamma)$, and it is also the strongly causal conformal boundary if $u \neq 0$ - if $u=0$, the strongly causal conformal boundary is empty (see Remark 9.8). Obviously, the invisible domain in $M_{\Lambda}(\Gamma)$ from $O$ is the quotient of the "intermediate region" $F_{1} \cap F_{2}$. 
It follows that $M_{\Lambda}(\Gamma)$ is an AdS-spacetime with a black-hole in the sense of Definition 1.2 if and only if $u \neq 0$.

Remark 10.2. The topology is very simple: $M_{\Lambda}$ is homeomorphic to the product of the annulus by $\mathbb{R}$. The same is true for the outer region and the black-hole, which are separated by a light-like annulus, the horizon.

\subsection{Splitting black holes}

Here, we consider the case where $\Lambda$ is splitting, i.e., two non-causally related points $(x, y)$. This case is fully detailed and described in Section 3.1. Figure 2 is still useful. The discussion above remains essentially the same, but now the conformal boundary of $E(\Lambda)$ has two connected components: $\Delta_{1}$ and $\Delta_{2}$. Hence, the invisible domain from their union is still $F_{1} \cap P_{1}$.

According to Section 5.2, in order to act properly on $E(\Lambda)$, the group $\Gamma$ must be as in the conical case: generated by a hyperbolic translation, or a hyperbolic-hyperbolic element. Hence, the conformal completion of the quotient is the quotient of these two diamond-shaped regions. The strongly causal conformal boundary is $\Delta_{1} \cup \Delta_{2}$, except if $\gamma$ is an hyperbolic translation, in which case the strongly causal boundary is empty.

When $\gamma$ is hyperbolic-hyperbolic, $M_{\Lambda}(\Gamma)$ contains a black-hole - the quotient of $F_{1} \cap F_{2}$ - isometric to the black-hole of the conical case.

The topological description is the same as in the conical case. But the horizon is not $C^{2}$, and the visible domain is not globally hyperbolic.

Remark 10.3. Something similar to what was discussed in Remark 10.1 appears: the conical spacetime $M_{\Lambda}(\Gamma)$ embeds isometrically in $M_{x y}(\Gamma)$ : hence, it is not maximal. This is a good reason for considering that conical case does not contain a black-hole, as in the "classical" literature. But observe that $M_{x y}(\Gamma)$ itself is not maximal too: when $u<v, M_{x y}(\Gamma)$ embeds in the spacetime $M^{D}(\Gamma)=\Gamma \backslash D(\Gamma)$, where $D(\Gamma)$ is the absolute causality domain (see Definition 4.8, Proposition 4.11, case (6)). Observe that $D(\Gamma)$ is an open domain in $\widetilde{\mathrm{AdS}}$ not contained in a affine domain. Actually, the proof of case (6) of Proposition 4.11 shows that $D(\Gamma)$ contains all the preimage in $\widetilde{\operatorname{AdS}}$ of $E_{x y}$, which are connected one to the other by regions (the "inner regions" with the conventions in $[4,5,11])$ which project in $\mathrm{AdS} \approx \mathrm{SL}(2, \mathbb{R})$ to the domain $0<b c<\frac{(\exp (u)-\exp (v))^{2}}{4 \exp (u+v)}$. Observe that these new regions have empty conformal boundaries: their closure intersects Ein $_{2}$ only along the union of two light-like geodesics. Hence, the conformal boundary of $D(\Gamma)$ is the preimage in $\widehat{\operatorname{Ein}}_{2}$ of all the preimage of $\Delta_{1} \cup \Delta_{2}$. 
In other words, let $A$ is an affine domain containing $E(\Lambda)$, and let $A_{i}$ be the infinite family of affine domains in $\widetilde{\mathrm{AdS}}$ such that $\widetilde{\mathrm{AdS}}$ is the union of the $\bar{A}_{i}$ (see $[7, \S 3.5]$ ). Every $\bar{A}_{i}$ contains a copy $E_{i}$ of $E(\Lambda)$. Moreover, $A$ can be selected so that the conformal boundary of $\bar{A}_{i}$ contains the conformal boundary $\Delta_{1}^{i} \cup \Delta_{2}^{i}$ of $E_{i}$. The inner regions connect every $\bar{A}_{i}$ to the following $\bar{A}_{i+1}$, allowing some causal curves to pass from $\bar{A}_{i}$ to $\bar{A}_{i+1}$. It is easy to show that, due to these connecting inner regions, $D(\Gamma)$ is entirely visible from its conformal boundary: it has no hole.

However, the quotient $M^{D}(\Gamma)$ considered in the literature devoted to BTZ black-holes (including [4]) has the typical spacetime containing a single nonstatic $(u \neq v)$ BTZ-black-hole. This requires one to pay attention to simple blocks $\bar{A}_{i} \cap D(\Gamma)$ individually, to consider the observers only in the boundary components of one of them, and to consider other blocks as being other parts of the universe which can be reached only by going through the horizon of the black-hole. Adopting this point of view, we observe that $M_{x y}(\Gamma)$ is therefore enough to give a picture of the considered black-hole.

Remark 10.4. This causal description is actually very similar to the description of black-holes in the maximal Kerr spacetime $M_{\mathrm{Kerr}}^{\max }$ (see the Introduction).

\subsection{The extreme black hole}

The case where $\Lambda$ is extreme is described in Section 3.2. In this case, the invisible domain is not contained in an affine domain, hence we need two successive domains, and $\Lambda$ must be considered as a closed subset of $\widehat{\operatorname{Ein}}_{2}$ (see figure 3). In this case, the conformal boundary is the extreme diamond $\Omega(x, y)$, and the entire $E(\Lambda)$ is visible from the boundary: there is no blackhole!

According to Section 5.1, the group $\Gamma$ must be contained in $A_{\text {hyp }}$ or $A_{\text {ext }}$, and since we want the action of $\Gamma$ on $E(\Lambda)$ to be causal, the case $\Gamma \subset A_{\text {hyp }}$ must be excluded (Section 5.1.2). Hence, the action of $\Gamma$ on $E(\Lambda)$ is free, properly discontinuous and strongly causal, except if it contains a parabolic translation (Section 5.1.1).

Anyway, as we have seen, $E(\Lambda)$ does not contain any black-hole. But we can use the same trick as for single non-static BTZ black-holes (Remark 10.3): consider the absolute causal domain $D(\Gamma)$. Observe that $D(\Gamma)=E(\Lambda)$ if $\Gamma$ is not cyclic (Theorem 8.2). Hence, this trick will apply only for cyclic subgroups of $A_{\text {ext }}$. Moreover, according to Proposition 4.11, case (3), the absolute causal domain of parabolic translations is trivial: we must exclude 
them. Finally, elements of $\Gamma$ are not hyperbolic translations since $\Gamma \subset A_{\text {hyp }}$ is excluded. Hence, $\Gamma$ must be generated by a parabolic-hyperbolic element. According to Proposition 4.11, case (7), the action of $\Gamma$ on $D(\Gamma)$ is free, properly discontinuous and proper.

We can reproduce nearly the same comment as in Remark 10.3: $D(\Gamma)$ has to be understood as a $\delta$-invariant subset of $\widetilde{\mathrm{AdS}}$, and the quotient space $M^{D}(\Gamma)$ is a union of "local universes". There is a small difference: the conformal boundary of every simple block is now connected (the other connect component "vanished"), and there is no intermediate region: the black-holes correspond to inner regions.

\subsection{Multi-black holes}

The last case we consider is the non-elementary case: $\Gamma$ is non-abelian and $\Lambda=\Lambda(\Gamma)$ is not elementary. The key point is to use [7, Proposition 8.50]: $E(\Lambda)$ is the union of the past and future globally hyperbolic cores, with the closed ends $\Omega(I)$ associated to gaps $I$ of $\Lambda$. Observe that the gaps in this case are not extreme ([7, Remark 8.25]); hence, the diamonds $\Omega(I)$ are not extreme. Moreover, since in this case the left and right morphisms are both faithful, the stabilizer of $\Omega(I)$ is generated by a hyperbolic-hyperbolic element.

It follows clearly that the connected components of the conformal boundary of $E(\Lambda)$ are precisely the diamonds $\Omega(I)$ : the only way to approach this conformal boundary is to enter a closed end. Observe that the closed end associated to a gap $I$ is isometric to the outer region of a conical spacetime. Moreover, if $\Lambda$ is a topological circle, then the conformal boundary is empty: there is no observer, no black-hole. Finally, the invisible domain from $\Omega(\Lambda)$ is precisely the future globally hyperbolic core $E\left(\Lambda^{+}\right)$.

Now, according to Theorem 5.1, and since every element of $\Gamma$ is hyperbolichyperbolic, the action of $\Gamma$ on $\Omega(\Lambda) \cup E(\Lambda)$ is free, properly discontinuous, and strongly causal (observe that it is true even if $\Lambda \neq \Lambda(\Gamma)$ ). The quotient spacetime $M_{\Lambda}(\Gamma)$ is then an AdS-spacetime with one black-hole: the (globally hyperbolic) quotient of $E\left(\Lambda^{+}\right)$by $\Gamma$.

Remark 10.5. There is an obvious 1-1 correspondence between the connected components of the conformal boundary of $M_{\Lambda}(\Gamma)$ and $\Gamma$-orbits of gaps, i.e., non-cuspidal boundary components of the surfaces $\Sigma_{\mathrm{L}} \approx \Sigma_{\mathrm{R}}$ (see Remark 6.3). 
Remark 10.6. According to Theorem 8.2, the trick used in Remark 10.3 in order to enlarge the spacetime by considering the absolute causal domain $D(\Gamma)$ gives nothing new: the quotient is the same spacetime.

Remark 10.7. Anyway, even if the "obvious" trick above does not work, many spacetimes $M_{\Lambda}(\Gamma)$ are not maximal AdS-spacetimes. Indeed, add to $E(\Lambda)$ a very small end $U$, such that the intersection $\partial U$ of the closure of $U$ with $\overline{\operatorname{Ein}}_{2} \approx \mathbb{R} P_{\mathrm{L}}^{1} \times \mathbb{R} P_{\mathrm{L}}^{1}$ is a small rectangle $I_{\mathrm{L}} \times I_{\mathrm{L}}$ around a point $x$ in the boundary of $\Omega(I)$ (for some gap $I$ ). Do not take as point $x$ one of the corner points of $\Omega(I)$. Then, if for some element $\gamma$ of $\Gamma$ the intersection $\partial U \cap \gamma \partial U$ is not empty, then $\gamma \Omega(I) \cap \Omega(I)$ is not empty. Then, $\gamma$ must belong to the cyclic subgroup $\Gamma_{I}$ of elements preserving the gap $I$ (see the proof of Theorem 5.1). Thus, $U$ can be chosen so that the intersection $\gamma U \cap U$ never happens. Then, the union of $E(\Lambda)$ with all the $\gamma U(\gamma \in \Gamma)$ is a $\Gamma$-invariant connected spacetime $E^{\prime}$ on which $\Gamma$ acts freely and properly discontinuously. Its quotient is a bigger spacetime $M^{\prime}$ in which $M_{\Lambda}(\Gamma)$ embeds isometrically.

Remark 10.8. Consider once more the spacetime $M^{\prime}$ constructed in the remark above. Let us prove that $M^{\prime}$ is strongly causal. There is no loss of generality in assuming that the point $x$ has been selected so that its (local) future does not meet $E(\Lambda) \cup \Omega(\Lambda)$. Then, no future-oriented causal curve starting from a point in $E^{\prime} \backslash E(\Lambda)$ that can enter $E(\Lambda)$ : we say that $E(\Lambda)$ is past-convex in $E^{\prime}$.

Let $y$ be an element of $E^{\prime}$. If $y$ belongs to $E(\Lambda)$, since $E(\Lambda)$ is causally convex and strongly causal, there is a neighborhood $V$ of $y$ which is not causally related to any non-trivial $\Gamma$-iterate of itself. Assume now that $y$ belongs to the end $U$ but not to $E(\Lambda)$. Observe that by construction $U$ meets $E(\Lambda)$ only in the closed end $E(I)$ associated to $I$. It follows that small neighborhoods of $y$ are contained in $U \backslash E(\Lambda)$. Any causal curve $c$ joining a point near $y$ to a point near $\gamma y$ with $\gamma \neq$ id must enter $E(\Lambda)$. Since $E(\Lambda)$ is past-convex in $E^{\prime}$ the causal curve $c$ must be past-oriented. But the same argument applied near $\gamma y$ shows that $c$ must be future-oriented.

This contradiction implies that the action of $\Gamma$ on $E^{\prime}$ is strongly causal. Therefore, $M^{\prime}$ is strongly causal.

It is quite clear that the conformal boundary of $M^{\prime}$ is the quotient by $\Gamma$ of the union of $\Omega(\Lambda)$ with the disjoint union of all the $\gamma \partial U$. Moreover, by causal convexity of $E(\Lambda) \cup \Omega(\Lambda)$, the strongly causal conformal boundary of $M^{\prime}$ contains as an open subset the conformal boundary of $M_{\Lambda}(\Gamma)$. We claim that the conformal boundary of $M_{\Lambda}(\Gamma)$ is actually a connected component of the strongly causal conformal boundary of $M^{\prime}$. Indeed, for every $y$ in $\partial U$ and in the boundary of $\Omega(I)$ in the Einstein space, for any neighborhood 
$V$ of $y$ in $\partial U$ and for any non-trivial element $\gamma$ of $\Gamma_{I}$ there are elements of $V \cap \Omega(I)$ causally related to elements of $\gamma V \cap \partial U$.

It follows quite easily that the strongly causal conformal boundary of $M^{\prime}$ is the quotient by $\Gamma$ of the union of $\Omega(\Lambda)$ with the disjoint union of all $\gamma(\partial U \backslash \bar{\Omega}(I))$, where $\gamma$ describes all $\Gamma$, and $\bar{\Omega}(I)$ is the closure in the Einstein space of $\Omega(I)$.

Points in $E^{\prime} \backslash E(\Lambda)$, i.e., elements of $\gamma U(\gamma \in \Gamma)$ are in the past of the strongly causal boundary of $E^{\prime}$. Hence, the black-hole in $M^{\prime}$ is contained in the black-hole of $M_{\Lambda}(\Gamma)$. Moreover, if the point $x$ has been selected in the past of $\Omega(I)$ (the proof of strong causality still applies, just replace pastconvex above by future-convex) then the black-hole in $M^{\prime}$ is equal to the black-hole in $M_{\Lambda}(\Gamma)$ : the part of the conformal boundary we added did not reveal any new point. But if the point $x$ has been selected in the future $\Omega(I)$ there is no general answer: the black-hole in $M^{\prime}$ might be strictly smaller than the black-hole in $M_{\Lambda}(\Gamma)$.

Remark 10.9. The example described in Remarks 10.7 and 10.8 shows that spacetimes with BTZ multi-black-holes are far from being maximal as spacetimes, even in the strongly causal category. But observe that these examples become forbidden if in the definition of spacetimes with blackholes we impose the following additional requirement: the strongly causal conformal boundary must be globally hyperbolic.

\subsection{Kerr-like coordinates}

We conclude with the presentation of the Kerr-like expression of the BTZ metric. This expression concerns the metric on the "outer regions", i.e., the ends. Hence we just have to consider the splitting and extreme cases.

\subsubsection{The Kerr-like metric on the outer region of a splitting spacetime}

In this case, $\Gamma$ is generated by $\gamma$ where, up to conjugacy,

$$
\gamma=\left(\gamma_{\mathrm{L}}, \gamma_{\mathrm{R}}\right)=(\exp (u \Delta), \exp (v \Delta)), \quad v \geq u>0
$$

The elements $x, y$ of $\Lambda$ must be the attractive and repulsive fixed points of $\gamma$. 
Define $r_{ \pm}\left(r_{+}>r_{-} \geq 0\right)$ by

$$
u=\pi\left(r_{+}-r_{-}\right), \quad v=\pi\left(r_{+}+r_{-}\right) .
$$

Then

$$
\gamma_{\mathrm{L}} g \gamma_{\mathrm{R}}^{-1}=\left(\begin{array}{ll}
a \exp \left(-2 \pi r_{-}\right) & b \exp \left(2 \pi r_{+}\right) \\
c \exp \left(-2 \pi r_{+}\right) & d \exp \left(2 \pi r_{-}\right)
\end{array}\right)
$$

Let $\left(x_{1}, x_{2}, y_{1}, y_{2}\right)$ be coordinates of $E$ for which $Q=d x_{1}^{2}+d x_{2}^{2}-d y_{1}^{2}-$ $d y_{2}^{2}$. Consider the identification

$$
\begin{aligned}
\mathrm{AdS} & \rightarrow \mathrm{SL}(2, \mathbb{R}), \\
\left(x_{1}, x_{2}, y_{1}, y_{2}\right) & \mapsto\left(\begin{array}{ll}
y_{1}+x_{1} & x_{2}+y_{2} \\
x_{2}-y_{2} & y_{1}-x_{1}
\end{array}\right) .
\end{aligned}
$$

Then, in the coordinates $\left(x_{1}, y_{1}, x_{2}, y_{2}\right)$, the matrix in $\mathrm{SO}(2,2)$ corresponding to $\gamma$ is

$$
\left(\begin{array}{cccc}
\cosh \left(2 \pi r_{-}\right) & -\sinh \left(2 \pi r_{-}\right) & 0 & 0 \\
-\sinh \left(2 \pi r_{-}\right) & \cosh \left(2 \pi r_{-}\right) & 0 & 0 \\
0 & 0 & \cosh \left(2 \pi r_{+}\right) & \sinh \left(2 \pi r_{+}\right) \\
0 & 0 & \sinh \left(2 \pi r_{+}\right) & \cosh \left(2 \pi r_{+}\right)
\end{array}\right) .
$$

The attractive and repulsive fixed points have coordinates $(0, \pm 1,0,1)$. Thus, the outer region is $\left\{\left|y_{1}\right|<x_{1},\left|x_{2}\right|<y_{2}\right\}$. A natural associated coordinate system on this domain is

$$
\begin{array}{ll}
x_{1}=\rho_{1} & \cosh (T), \\
y_{1}=\rho_{1} & \sinh (T), \\
x_{2}=\rho_{2} & \sinh (\phi), \\
y_{2}=\rho_{2} & \cosh (\phi),
\end{array}
$$

with $\rho_{1}^{2}=\rho_{2}^{2}-1$. The AdS-metric $d x_{1}^{2}+d x_{2}^{2}-d y_{1}^{2}-d y_{1}^{2}$ in the coordinates $\left(T, \phi, \rho=\rho_{2}\right)$ is

$$
\frac{1}{\rho^{2}-1} d \rho^{2}+\rho^{2} d \phi^{2}-\left(\rho^{2}-1\right) d T^{2}
$$

Observe that $\phi, T$ may have any real value, and that $\rho$ takes value in ] $1,+\infty[$.

The action of $\gamma$ in the coordinates $\left(\phi, T, \rho_{1}, \rho_{2}\right)$ is simply the translation by $2 \pi r_{+}$on $\phi$ and by $-2 \pi r_{-}$on $T$. Hence, it preserves the function $t=$ $\frac{r_{-} \phi+r_{+} T}{r_{+}^{2}-r_{-}^{2}}$, and adds to $\varphi=\frac{r_{+} \phi+r_{-} T}{r_{+}^{2}-r_{-}^{2}}$ the term $2 \pi$. Therefore, we introduce the coordinates $(t, \varphi)$ instead of $(T, \phi): \varphi$ is considered as a polar coordinate, the action by $\gamma$ being represented by $\varphi \rightarrow \varphi+2 \pi$, the other coordinates $(t, \rho)$ 
remaining unchanged. Actually, replace $\rho$ by the coordinate $r$ defined by $\rho^{2}=\frac{r^{2}-r_{-}^{2}}{r_{+}^{2}-r_{-}^{2}}$. Then, the AdS-metric becomes

$$
-N(r) d t^{2}+N(r)^{-1} d r^{2}+r^{2}\left(d \varphi+\frac{J}{2 r^{2}} d t\right)^{2}
$$

where

$$
\begin{aligned}
J & =-2 r_{-} r_{+}, \\
N(r) & =\frac{\left(r^{2}-r_{+}^{2}\right)\left(r^{2}-r_{-}^{2}\right)}{r^{2}} .
\end{aligned}
$$

The coordinates $(\varphi, t, r)$ are the Kerr-like coordinates. Considering $\varphi$ as defined modulo $2 \pi$, it provides an expression of the outer region of $M_{x y}(\Gamma)$. The analogy with the Kerr metric is striking if we observe $N(r)=r^{2}-M+$ $\left(\frac{J}{2 r}\right)^{2}$, where $M=r_{+}^{2}+r_{-}^{2}$.

Remark 10.10. In the Kerr-like coordinates, the level sets of the time function $t$ are not homogeneous. The stabilizer of the outer region is $A_{\text {hyp }}$, the orbits of which in the outer region are time-like. Observe that this action of $A_{\text {hyp }}$ is an action by translations on the coordinates $(t, \varphi)$. Actually, the (scalar) curvature of the level sets of $t$ is not constant (except if $r_{-}=0$ ). What is the specific geometric feature of these level sets?

A remarkable fact is that the level sets $\left\{t=t_{0}\right\}$ are maximal, i.e., have zero mean curvature. A quick computation is as follows: parameterize the level set $S_{0}=\left\{t=t_{0}\right\}$ by parameters $\eta, \varphi$, where $\rho=\cosh (\eta)$ :

$$
\begin{aligned}
& x_{1}=\sinh (\eta) \cosh (T), \\
& y_{1}=\sinh (\eta) \sinh (T), \\
& x_{2}=\cosh (\eta) \sinh (\phi), \\
& y_{2}=\cosh (\eta) \cosh (\phi),
\end{aligned}
$$

with $T=r_{+} t_{0}-r_{-} \varphi, \phi=r_{+} \varphi-r_{-} t_{0}$. Let $p$ be a point of $S_{0}$ of coordinates $(\eta, \varphi)$. Identify the tangent space to $\operatorname{AdS}$ at $p$ with the $Q$-orthogonal $p^{\perp}$ in $E$. Then the tangent vectors of $S_{0}$ at $p$ are generated by

$$
\partial_{\eta}=\left(\begin{array}{c}
\cosh (\eta) \cosh (T) \\
\cosh (\eta) \sinh (T) \\
\sinh (\eta) \sinh (\phi) \\
\sinh (\eta) \cosh (\phi)
\end{array}\right), \quad \partial_{\varphi}=\left(\begin{array}{c}
-r_{-} \sinh (\eta) \sinh (T) \\
-r_{-} \sinh (\eta) \cosh (T) \\
r_{+} \cosh (\eta) \cosh (\phi) \\
r_{+} \cosh (\eta) \sinh (\phi)
\end{array}\right) .
$$


The future-oriented normal $n_{0}$ to $S_{0}$ at $p$ is the following vector $n$, divided by the square root of the opposite of its norm, which is $r_{-}^{2} \sinh (\eta)^{2}-$ $r_{+}^{2} \cosh (\eta)^{2}=-r^{2}$ :

$$
n=\left(\begin{array}{c}
r_{+} \cosh (\eta) \sinh (T) \\
r_{+} \cosh (\eta) \cosh (T) \\
-r_{-} \sinh (\eta) \cosh (\phi) \\
-r_{-} \sinh (\eta) \sinh (\phi)
\end{array}\right)
$$

Now, the second fundamental form of $S_{0}$ on a tangent vector field $X$ at $p$ is obtained by computing $\left\langle n_{0} \mid \nabla_{X} X\right\rangle$. But the Levi-Civita connection of AdS is just the orthogonal projection on TAdS of the usual flat connection of $E$. Hence, the (extrinsic) curvatures of the curves $\{\varphi=C t e\}$ and $\{\eta=C t e\}$ are the $Q$-scalar products with $n_{0}$ of the following vectors:

$$
\partial_{\eta \eta}=\left(\begin{array}{c}
\sinh (\eta) \cosh (T) \\
\sinh (\eta) \sinh (T) \\
\cosh (\eta) \sinh (\phi) \\
\cosh (\eta) \cosh (\phi)
\end{array}\right)=p, \quad \partial_{\varphi \varphi}=\left(\begin{array}{c}
r_{-}^{2} \sinh (\eta) \cosh (T) \\
r_{-}^{2} \sinh (\eta) \sinh (T) \\
r_{+}^{2} \cosh (\eta) \sinh (\phi) \\
r_{+}^{2} \cosh (\eta) \cosh (\phi)
\end{array}\right) .
$$

These scalar products are null. Since the curves $\{\eta=C t e\}$ and $\{\varphi=C t e\}$ are everywhere orthogonal, the mean curvature of $S_{0}$ at $p$ is thus 0 (but these curves do not define the principal directions if $\left.r_{-} \neq 0\right)$. Observe that $\{\varphi=C t e\}$ is actually a space-like geodesic in AdS.

Moreover, $\left\langle n \mid \partial_{\eta \varphi}\right\rangle=r_{-} r_{+}$. It follows that the second fundamental form is II $=r^{-1} r_{-} r_{+} d \eta d \varphi$. The pair $\left(\partial_{\eta}, r^{-1} \partial_{\varphi}\right)$ is an orthonormal basis of the tangent space at $p$. Hence, the Gauss curvature of $S_{0}$ is

$$
\frac{r_{-}^{2} r_{+}^{2}}{4 r^{4}}
$$

\subsubsection{The Kerr-like metric on the outer region of an extreme spacetime}

Observe that the Kerr-like metric remains meaningful when $r_{-}=r_{+}$, even if the coordinate transformations considered in the previous section are not valid anymore. Let $E^{\prime}\left(r_{+}\right)$be (simply connected) lorentzian manifold consisting of $\left.\mathbb{R}^{2} \times\right] r_{+},+\infty[$ with coordinates $(t, \varphi, \rho)$, equipped with the metric

$$
-N(r) d t^{2}+N(r)^{-1} d r^{2}+r^{2}\left(d \varphi+\frac{J}{2 r^{2}} d t\right)^{2},
$$


where

$$
\begin{aligned}
J & =-2 r_{+}^{2} \\
N(r) & =\frac{\left(r^{2}-r_{+}^{2}\right)^{2}}{r^{2}}=r^{2}-2 r_{+}^{2}+\left(\frac{J}{2 r}\right)^{2}
\end{aligned}
$$

$M^{\prime}\left(r_{+}\right)$is the quotient of $E^{\prime}\left(r_{+}\right)$by the translation $\varphi \rightarrow \varphi+2 \pi$, the other coordinates remaining the same. We want to prove that $E^{\prime}\left(r_{+}\right)$is isometric to the outer region of an extreme black-hole as defined in Section 10.3.

The sectional curvature of the Kerr-like metric for $r_{-}=r_{+}-\epsilon$ is -1 for any $\epsilon$; it then remains true at the limit $\epsilon=0$. Hence, $E^{\prime}\left(r_{+}\right)$and $M^{\prime}\left(r_{+}\right)$ are locally AdS. Observe also that $\partial_{\varphi}$ and $\partial_{t}$ generate a rank 2 abelian Lie algebra of Killing vector fields. This is the pull-back by the developing map of an abelian Lie algebra $\mathcal{A}\left(r_{+}\right)$of Killing vector fields on AdS. This Lie algebra is of course a limit of algebras $\mathcal{A}\left(r_{+}, r_{+}-\epsilon\right)$ which are all Lie algebras of subgroups conjugate to $A_{\text {hyp }}$. It follows easily that $\mathcal{A}\left(r_{+}\right)$is conjugate either to the Lie algebra of $A_{\text {hyp }}$, or to the Lie algebra of $A_{\text {ext }}$. A quick calculus shows that $\partial_{\varphi}+\partial_{t}$ is an everywhere-light-like Killing vector field: it excludes the hyperbolic-hyperbolic case $A_{\text {hyp }}$; hence, up to conjugacy, the isometry group generated by $\partial_{t}$ and $\partial_{\varphi}$ is $A_{\text {ext }}$.

Parameterize a line $\{t=C t e, \rho=C t e\}$ by $\varphi$, and compute $\left\langle\partial_{\varphi \varphi} \mid p\right\rangle=$ $-r^{2}$ (once more, we can compute for $r_{-}=r_{+}-\epsilon$ ). Hence, the orbits of the translations on the $\varphi$-coordinate are not geodesic. It follows that they are not hyperbolic translations: they are hyperbolic-parabolic. In other words, the monodromy of the translation $\varphi \rightarrow \varphi+2 \pi$ is conjugate to $\gamma^{\prime}=$ $\left(\exp \left(2 \pi r_{0} \Delta\right), \exp (H)\right)$, for some $r_{0}>0$. Actually, $r_{0}=r_{+}$: indeed, for $r_{-}=$ $r_{+}-\epsilon$, the left component of the monodromy of $\varphi \rightarrow \varphi+2 \pi$ is conjugate to $\exp \left(2 \pi r_{+}\right)$, it remains true at the limit $\epsilon=0$ by continuity of the monodromy under deformations of the AdS-structure. Hence $\gamma^{\prime}=\gamma$.

We observe now that $\partial_{\varphi}$ is space-like on $E^{\prime}\left(r_{+}\right)$. Hence, the image of the developing map of $E^{\prime}\left(r_{+}\right)$is contained in $D(\gamma)$. The curve $c(r)=(t=0, \varphi=$ $0, r)$ is a geodesic (for example, the study in the preceding section shows that it is true in the case $r_{-}<r_{+}$, and the case $r_{-}=r_{+}$is a limit case). Moreover, the length between two points $c(r), c\left(r^{\prime}\right)$ is $\frac{1}{2} \log \left(\frac{r^{\prime 2}-r_{+}^{2}}{r^{2}-r_{+}^{2}}\right): c$ is a complete space-like geodesic. According to the description of absolute causal domains of hyperbolic-parabolic elements (case (7) of Proposition 4.11), the complete space-like geodesics entirely contained in $D(\gamma)$ must actually lie in $C(\gamma)$, which is the outer region $E$ of the extreme black-hole associated to

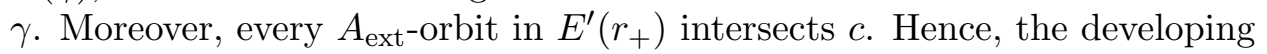
image of $E^{\prime}\left(r_{+}\right)$is contained in $E$. The action of $A_{\text {ext }}$ on $E$ is free: it 
follows that the restriction of the developing map $\mathcal{D}$ to any $A_{\text {ext-orbit in }}$ $E^{\prime}\left(r_{+}\right)$is a homeomorphism onto an entire $A_{\text {ext }}$-orbit in $E$. Finally, the restriction of $c$ is injective, with image a complete space-like geodesic $\bar{c}$ in $E$. Every $A_{\text {ext }}$-orbit in $E$ intersects $\bar{c}$. It follows that $\mathcal{D}$ is an isometry between $E^{\prime}\left(r_{+}\right)$and $E$ : the coordinates $(t, \varphi, r)$ parameterize the entire outer region $E$.

Remark 10.11. The limit " $r=r_{+}$" is a light-like segment $\tau$ in $\partial$ AdS. It follows that the time levels $\{t=C t e\}$ are closed in AdS. Their closure in AdS $\cup \partial A d S$ intersects $\partial \mathrm{AdS}$ along an achronal topological circle which contains the light-like segment $\tau$, and is space-like outside $\tau$. Compare this situation with the example described in [19, p. 45].

Of course, these level sets have zero mean curvature since it is true in the non-extreme case $r_{-}<r_{+}$. Furthermore, they have Gauss curvature $r_{+}^{4} / 4 r^{4}$.

\section{Acknowledgments}

L. Freidel introduced me to the realm of BTZ black-holes and their physical motivations. He initiated my special interest to Lorentzian geometry with constant curvature. F. Béguin, C. Frances, A. Zeghib have participated in the early stage of the conception of this work in the mathematical and geometrical understanding of BTZ-black holes and related questions. P. Saadé has drawn the pictures.

The work is supported by CNRS, ACI "Structures géométriques et Trous Noirs".

\section{References}

[1] S. Aminneborg, I. Bengtsson, S. Holst and P. Peldan, Making antide Sitter black holes, Class. Quant. Grav. 13 (10) (1996); 2707-2714; arXiv:gr-qc/9604005.

[2] S. Aminneborg, I. Bengtsson, D.R. Brill, S. Holst and P. Peldan, Black holes and wormholes in $2+1$ dimensions, Class. Quant. Grav. 15 (3) (1998), 627-644; arXiv:gr-qc/9707036.

[3] S. Aminneborg, I. Bengtsson and S. Holst, A spinning anti-de Sitter wormhole, Class. Quant. Grav. 16 (2) (1999), 363-382; arXiv:grqc/9805028 v2. 
[4] M. Bañados, C. Teitelboim and J. Zanelli, The black hole in threedimensional spacetime, Phys. Rev. Lett. 69 (13) (1992), 1849-1851; arXiv:hep-th/9204099.

[5] M. Bañados, M. Henneaux, C. Teitelboim and J. Zanelli, Geometry of the $2+1$ black hole, Phys. Rev. D (3) 48 (4) (1993), 1506-1525; arXiv:gr-qc/9302012.

[6] T. Barbot, Variétés affines radiales de dimension 3, Bull. Soc. Math. France 128 (2000), 347-389.

[7] T. Barbot, Causal properties of AdS-isometry groups I: causal actions and limit sets, Adv. Theor. Math. Phys. 12 (1) (2008), 1-66; arXiv:math.GT/0509552.

[8] J.K. Beem, P.E. Ehrlich and K.L. Easley, Global Lorentzian geometry, 2nd edn, Monographs and Textbooks in Pure and Applied Mathematics, 202, Marcel Dekker, New York, 1996.

[9] R. Benedetti and F. Bonsante, Canonical Wick rotations in 3dimensional gravity, Memoirs of the AMS, arXiv:math.DG/0508485.

[10] D.R. Brill, Multi-black-hole geometries in $(2+1)$-dimensional gravity, Phys. Rev. D (3) 53 (8) (1996), R4133-R4137; arXiv:gr-qc/9511022.

[11] D.R. Brill, Black holes and wormholes in $2+1$ dimensions, 'Mathematical and Quantum Aspects of Relativity and Cosmology (Pythagoreon, 1998)'; Lecture Notes in Phys., 537, Springer, Berlin, 2000, 143-179; arXiv:gr-qc/9904083.

[12] S. DeDeo and J.R. Gott III, An eternal time machine in $2+1$ dimensional anti-de Sitter space, Phys. Rev. D66 (2002), 084020; Erratum ibid. D67 (2003) 069902; arXiv:gr-qc/0212118.

[13] C. Frances, The conformal boundary of anti-de Sitter spacetimes, Actes du Colloque 'AdS/CFT Correspondence', Strasbourg, 10-13 September 2003.

[14] R. Fricke and K. Klein, Vorlesungen des Automorphen Funktionen, Vols I and II, Teubner, Leipzig, 1897/1912.

[15] A. García - Parrado and J.M.M. Senovilla, Causal structures and causal boundaries, Class. Quant. Grav. 22 (9) (2005), R1-R84; arXiv:grqc/0501069.

[16] W. Goldman, The modular group action on real SL(2)-characters of a one-holed torus, Geom. Topol. 7 (2003), 443-486.

[17] P. Hajicek, Extensions of the Taub and NUT spaces and extensions of their tangent bundle, Commun. Math. Phys. 17 (1970), 109-126.

[18] H.J. Matschull, Black hole creation in $2+1$ dimensions, Class. Quant. Grav. 16 (1999), 1069-1095; arXiv:gr-qc/9809087. 
[19] G. Mess, Lorentz spacetimes of constant curvature, preprint IHES/M/90/28, 1990.

[20] C.W. Misner, Taub-NUT space as a counterexample to almost anything, Relativity Theory and Astrophysics (AMS Providence, RI), Lect. Appl. Math. 8 (1967), 160-169.

[21] C.W. Misner, K.S. Thorne and J.A. Wheeler, Gravitation, Freeman, San Fransisco, 1973.

[22] B. O'Neill, The geometry of Kerr black holes, A.K. Peters, Wellesley, MA, 1995.

[23] F. Salein, Variétés anti-de Sitter de dimension 3 possédant un champ de Killing non trivial, C.R. Acad. Sci. Paris Sér. I Math. 324 (1997), 525-530. 
